\title{
REVIEWS
}

TUMOUR IMMUNOLOGY

\section{Cancer despite immunosurveillance: immunoselection and immunosubversion}

\section{Laurence Zitvogel ${ }^{*}$, Antoine Tesniere ${ }^{\ddagger}$ and Guido Kroemer ${ }^{\ddagger}$}

Abstract | Numerous innate and adaptive immune effector cells and molecules participate in the recognition and destruction of cancer cells, a process that is known as cancer immunosurveillance. But cancer cells avoid such immunosurveillance through the outgrowth of poorly immunogenic tumour-cell variants (immunoselection) and through subversion of the immune system (immunosubversion). At the early stages of carcinogenesis, cell-intrinsic barriers to tumour development seem to be associated with stimulation of an active antitumour immune response, whereas overt tumour development seems to correlate with changes in the immunogenic properties of tumour cells. The permanent success of treatments for cancer might depend on using immunogenic chemotherapy to re-establish antitumour immune responses.

Angiogenesis

The development of new blood vessels from existing blood vessels. It is frequently associated with tumour development and inflammation

Natural killer T cells (NKT cells). A heterogeneous subset of $T$ cells that are characterized by the co-expression of semi-invariant T-cell receptor $\alpha$-chains together with NK-cell markers.

* U805 Institut National de la Santé et de la Recherche Médicale, Faculté de Médecine Paris-SudUniversité Paris XI, Institut Gustave-Roussy, 39 rue Camille-Desmoulins, F-94805 Villejuif, France. ${ }^{\ddagger}$ Centre National de la Recherche Scientifique FRE29239, Institut Gustave-Roussy, 39 rue Camille-Desmoulins, F-94805 Villejuif, France. Correspondence to G.K. e-mail: kroemer@igr.fr doi:10.1038/nri1936 Published online 15 September 2006
It is well established and generally accepted that six cellintrinsic (cell-autonomous) phenomena determine early oncogenesis. Cancer cells characteristically provide their own growth signals, ignore growth-inhibitory signals, avoid cell death, replicate without limits, sustain angiogenesis, and invade tissues through basement membranes and capillary walls ${ }^{1}$. In addition, as recently proposed by Schreiber and colleagues ${ }^{2,3}$, avoidance of immunosurveillance might be the seventh hallmark of cancer. Cancer cells escape innate and adaptive immune responses - cancer immunosurveillance - by immunoselection (that is, selection of non-immunogenic tumour-cell variants, a process that is also known as immunoediting) or by immunosubversion (that is, active suppression of the immune response) $)^{4}$.

The importance of cancer immunosurveillance is still controversial, especially among nonimmunologists and, in particular, among oncologists, who often think in cell-intrinsic terms. Although evidence that immunosurveillance has a fundamental role in cancer development and anticancer therapy has been accumulating in recent years, these findings have had little impact on the accepted theories of multistep carcinogenesis and have not influenced the way in which anticancer therapies are conceived and applied in the clinic. This Review summarizes recent studies that elucidate the numerous links between cell-extrinsic (immune-mediated) and cell-intrinsic mechanisms of suppression of tumours (FIG. 1), both of which need to be subverted for cancer to develop. We also suggest that the seventh hallmark of cancer (that is, avoidance of immunosurveillance) is mechanistically linked to the six established cell-intrinsic characteristics of cancer cells.

\section{Cancer immunosurveillance}

The concept of cancer immunosurveillance predicts that the immune system can recognize precursors of cancer and, in most cases, destroy these precursors before they become clinically apparent (FIG. 2). It is well established that mice that lack essential components of the innate or adaptive immune system are more susceptible to the development of spontaneous or chemically induced tumours. This is the case for animals that lack the following components: recombination-activating gene 2 (RAG2), lack of which results in the absence of T cells, B cells and natural killer T (NKT) cells; the $\gamma$-chain of the T-cell receptor (TCR $\gamma$ ), lack of which results in the absence of $\gamma \delta$ T cells; TCR $\beta$ and TCR $\gamma$, lack of which results in the absence of $\alpha \beta$ and $\gamma \delta \mathrm{T}$ cells; the joining gene segment J $\alpha 281$, lack of which reduces the number of invariant NKT cells expressing Vo14-J $\alpha 281$ containing TCRs; interferon- $\gamma$ (IFN $\gamma$ ) receptor 1 (REF. 5); signal transducer and activator of transcription 1 (STAT1), a transcription factor that is required for IFN $\gamma$-induced signalling; perforin; or tumour-necrosis 


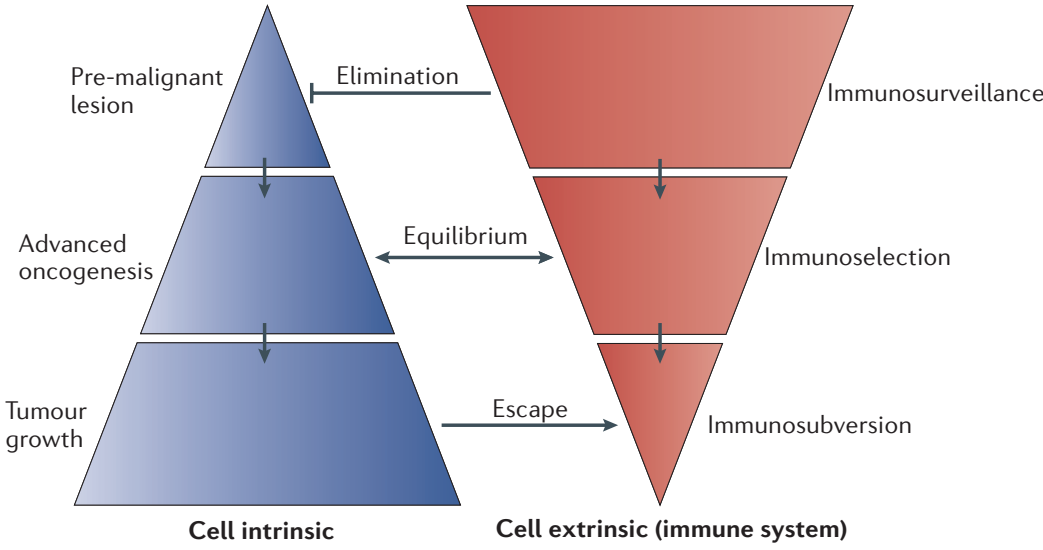

Figure 1 | Relationship between cell-intrinsic and cell-extrinsic aspects of tumour progression. This figure illustrates the central concept that multistep carcinogenesis results from crosstalk of cancer-cell-intrinsic factors and host immune system (cell-extrinsic) effects.

\section{Perforin}

A component of cytolytic granules that participates in the permeabilization of plasma membranes, allowing granzymes and other cytotoxic components to enter target cells

\section{NKG2D}

(Natural-killer group 2, member D). A lectin-type activating receptor that is encoded by the NK complex and is expressed at the surface of NK cells, NKT cells, $\gamma \delta$ T cells and some cytolytic CD8 $\alpha \beta$ T cells. The ligands for NKC2D are MHC-class-Ipolypeptide-related sequence $\mathrm{A}$ (MICA) and $\mathrm{MICB}$ in humans, and retinoic acid early transcript 1 (RAE1) and $\mathrm{H} 60$ in mice. Such ligands are generally expressed at the surface of infected, stressed or transformed cells.

IFN-producing killer DC (IKDC). A dendritic cell (DC) that expresses markers of both natural killer cells and B cells but lacks markers of plasmacytoid DCs and T cells, as well as co-stimulatory molecules. These DCs have been isolated only in mouse models. They respond to a large variety of tumour cells by producing interferon- $\gamma$ (IFN $\gamma$ ), and they kill these tumour cells in the absence of exogenous stimulation

Pre-malignant

An intermediate step before full transformation into a tumour cell has occurred. factor (TNF)-related apoptosis-inducing ligand (TRAIL) (reviewed in REF. 4).

Such genetic experiments are supported by studies in which antibodies are used to deplete natural killer (NK) cells and NKT cells or to neutralize TRAIL ${ }^{6}$ or the activating receptor NKG2D (NK group 2, member D) ${ }^{7}$. By contrast, an immunostimulatory regimen designed to increase the number of NK cells and invariant NKT cells can reduce the development of malignant disease in mouse models ${ }^{8}$. Furthermore, a novel immunostimulatory regimen that comprises imatinib mesylate (Gleevec or Glivec; Novartis) plus interleukin-2 (IL-2) stimulates antitumour immune responses; in particular, this regimen elicits the expansion of a novel subset of dendritic cells (DCs), IFN-producing killer DCs (IKDCs), that can mediate the regression of tumours in vivo in a TRAIL-dependent manner ${ }^{9}$. In summary, from the current scientific literature, it is possible to identify several cell types and a range of effector molecules that are involved in cancer immunosurveillance (FIG. 2).

If immunosurveillance has an important role in the suppression of tumours, then it would be expected that patients with pre-malignant or early cancerous lesions mount vigorous immune responses. There are, indeed, several lines of evidence that indicate that this might be the case. For example, patients with monoclonal gammopathy, which is pre-malignant, mount strong $\mathrm{T}$-cell responses to autologous pre-malignant $\mathrm{B}$ cells, whereas no such responses are found in patients with multiple myeloma, which is malignant ${ }^{10}$. In addition, the bone marrow of patients with operable breast cancer contains $\mathrm{CD}^{+} \mathrm{T}$ cells specific for peptides derived from the breast-cancer-associated proteins mucin-1 (MUC1) and ERBB2 (also known as HER2 and NEU), and such $\mathrm{T}$ cells can mediate the regression of autologous human tumours that have been transplanted into immunodeficient non-obese diabetic mice ${ }^{11}$. Similar results have been reported for patients with pancreatic cancer; these patients have bone-marrow cells that can reject tumour xenotransplants when transferred to the recipient animals ${ }^{12}$.

These examples and others that are discussed later indicate that, although tumours induce at least transient immune responses, cancer can still develop. Such cellular immune responses might be too inefficient to prevent the development of cancer either because tumour cells that evade the immune response are selected (known as immunoselection) or because tumour-antigenspecific immune tolerance is induced ${ }^{13}$. Nevertheless, tumour-specific antibody responses (also known as antibody signatures) can be used to detect cancers such as prostate cancer at early stages ${ }^{14}$. Moreover, antibodies specific for cyclin B1 (REF. 15) or the tumour-suppressor protein p53 (REF. 16) might be useful for detecting cancer at early stages. At present, serum cyclin-B1- and MUC1specific antibodies are being assessed for their potential as prognostic biomarkers ${ }^{17}$.

Figure 2 |Cancer immunosurveillance. a $\mid \mathrm{CD} 8^{+}$cytotoxic $\mathrm{T}$ lymphocytes (CTLs) recognize and kill stromal and/or tumour cells in an MHC-restricted and perforin-dependent manner. In parallel, they secrete the anti-angiogenic cytokine interferon- $\gamma(\mathrm{IFN} \gamma)$. $\mathbf{b} \mid$ Activated $\mathrm{CD} 4^{+} \mathrm{T}$ cells recognize tumour-infiltrating macrophages in an MHC-class-II-dependent manner, converting interleukin-10 (IL-10)-producing M1 macrophages into IFN $\gamma$-producing M2 macrophages. Activated CD4 $4^{+}$Thelper $1\left(T_{H} 1\right)$ cells can also release IFN $\gamma$, which inhibits angiogenesis. $T_{H} 2$ cells produce IL-4 and block neo-angiogenesis indirectly, through an effect on stromal fibroblasts. c| IFN-producing killer dendritic cells (IKDCs) kill tumour cells in a TRAIL (tumour-necrosis factor (TNF)-related apoptosis-inducing ligand)and perforin-dependent manner. IKDCs are also a major source of IFN $\gamma$. IKDCs might also cross-present tumour antigens to T cells. $\mathbf{d}$ | During chronic myeloid leukaemia, natural killer (NK) cells can be activated in an NKG2D (NK group 2, member D)-dependent manner by dendritic cells (DCs) with a BCR-ABL (breakpoint-cluster region fused with Abelson leukaemia-virus protein) translocation. During chronic inflammation and in the presence of IL-4 and IL-13, DCs upregulate TREM2 (triggering receptor expressed on myeloid cells 2), which is required for NK-cell triggering. Inhibitors of KIT promote NK-cell activation through cell-cell contact between DCs and NK cells. e | Natural killer T (NKT) cells recognize glycolipids bound to CD1d at the surface of antigen-presenting cells (APCs). Activated NKT cells secrete IFN $\gamma$ in a CD40- and IL-12-dependent manner and can lyse tumour cells though an MHC-unrestricted, TRAILor perforin-dependent pathway. $\mathbf{f}$ | Tumour cells or APCs present the $\mathrm{F}_{1}$-ATPase-apolipoprotein-A complex or phosphoantigens to $\gamma \delta \mathrm{T}$ cells with a $\mathrm{V} \gamma 9 \mathrm{~V} \delta 2$-containing T-cell receptor (TCR), and these $\gamma \delta \mathrm{T}$ cells produce cytokines or kill tumour cells in an NKG2D-dependent manner. $\mathbf{g}$ |Tumour-specific antibodies could have a tumoricidal role by promoting antiproliferative effects directly or by inducing complement-mediated lysis or antibody-dependent cytotoxicity by NK cells and macrophages expressing receptors for $\operatorname{lgG}(\mathrm{Fc} \gamma \mathrm{Rs})$. Question marks denote pathways that need to be confirmed by further studies. BrHDP, bromohydrin diphosphate (also known as BrHPP); CD40L, CD40 ligand; IDP, isopentenyl diphosphate (also known as IPP); RAE1, retinoic acid early transcript 1. 
Monoclonal gammopathy A tumour (most of which develop into multiple myelomas) that is derived from $B$ cells and produces one type of monoclonal antibody, which can be detected in the serum.
In numerous cancers, the presence of tumourinfiltrating T lymphocytes (TILs) is a useful prognostic marker. This applies, in particular, to melanoma, ovarian carcinoma and colon carcinoma ${ }^{18}$. In patients with colorectal carcinoma, the presence of mRNA encoding molecules expressed by effector T helper 1 $\left(T_{H} 1\right)$ cells (such as CD8 $\alpha$, granzyme B, granulysin, T-bet, IFN $\gamma$ and IFN-regulatory factor 1) and effector memory $\mathrm{T}$ cells correlates with reduced metastatic invasion and increased survival of the patient ${ }^{19}$. By contrast, in patients with renal-cell carcinoma, the presence of a polymorphism in the IL-4 receptor that results in increased IL-4-receptor signalling and a bias towards $\mathrm{T}_{\mathrm{H}}$ 2-cell responses is an independent indicator of adverse prognosis for the patient ${ }^{20}$. In patients with ovarian carcinoma or melanoma, the presence of $\mathrm{CD} 4^{+} \mathrm{CD} 25^{+}$ a

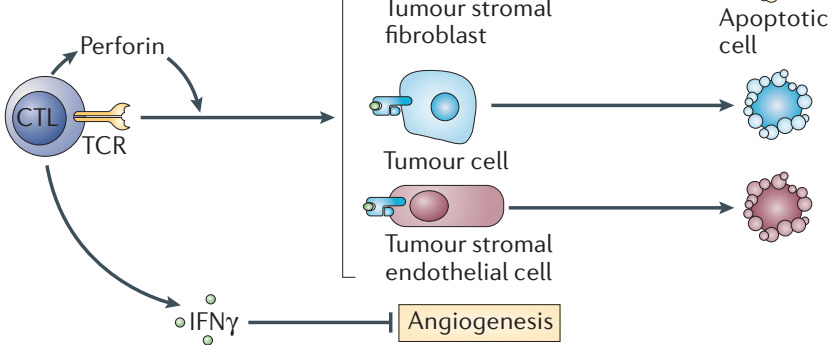

c


f $\quad$ BrHDP, IDP and

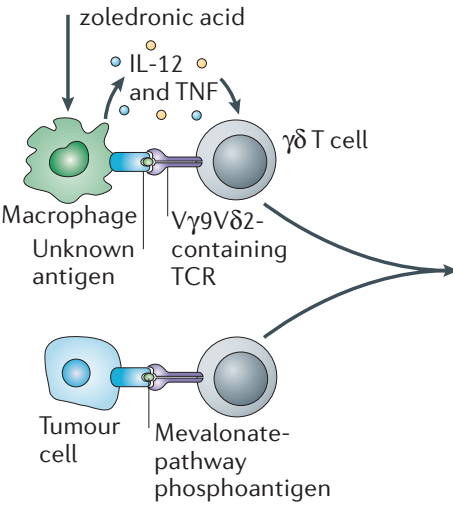

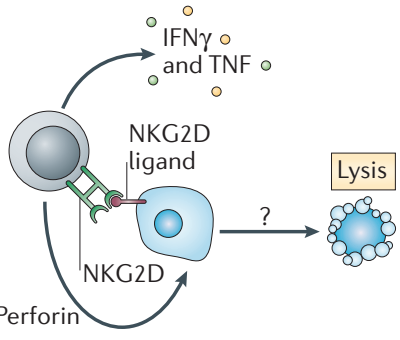

Perforin

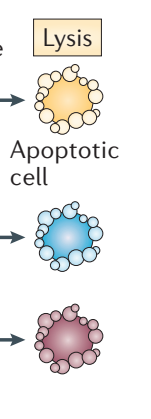

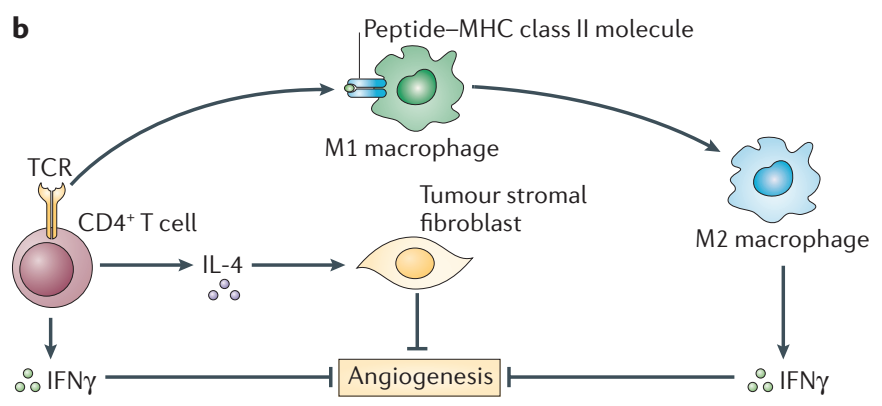

d
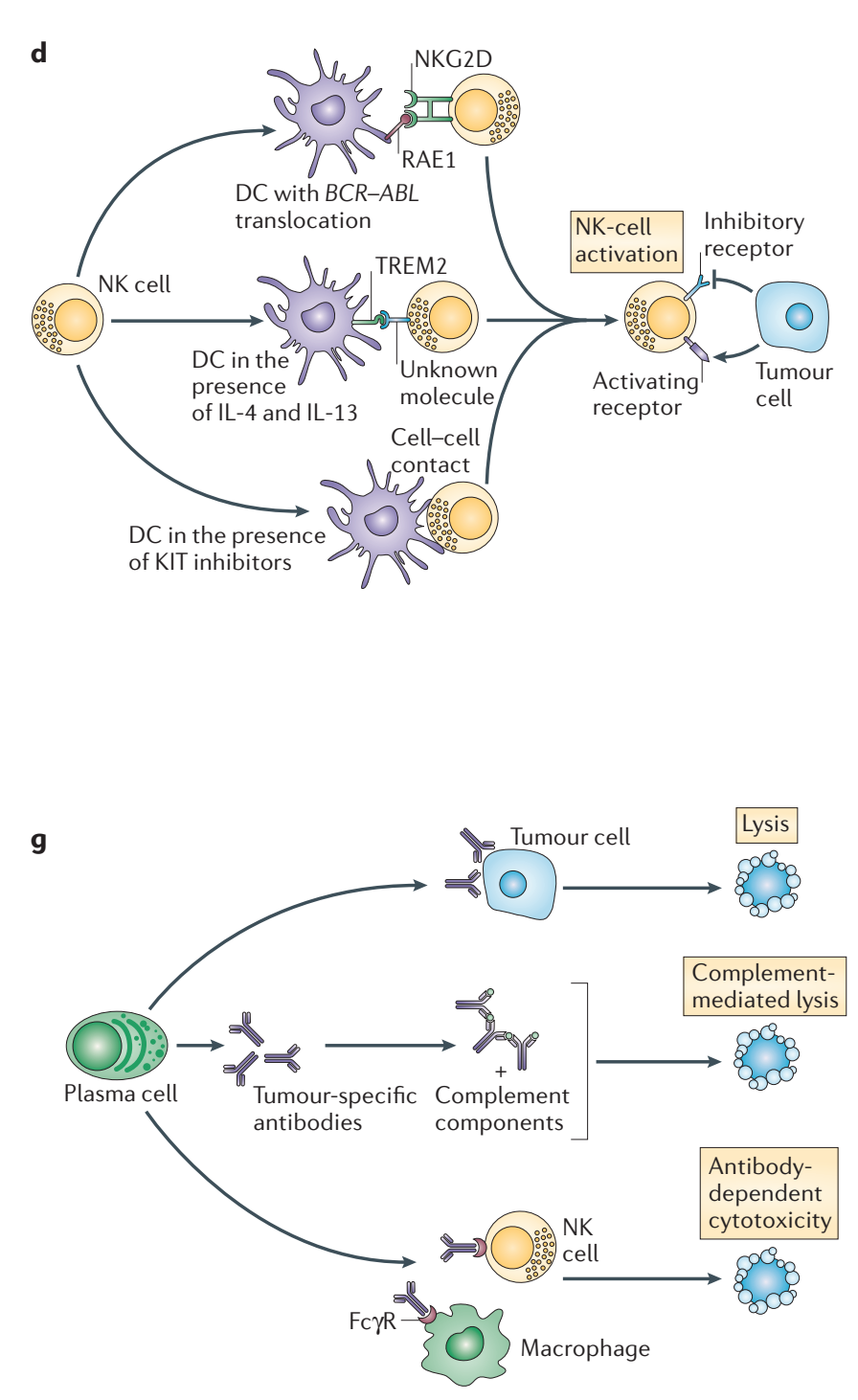

$$
\text { im }
$$


Tumour-suppressor protein A protein encoded by a gene that, when eliminated or inactivated, allows the development of cancer. These proteins often determine cellcycle checkpoints or facilitate the induction of apoptosis

p53

An important transcription factor that is activated by many genotoxic insults and induces cellular senescence or apoptosis. The gene that encodes p53 is frequently mutated or functionally inactivated in cancer cells.

T helper 1 cell

$\left(\mathrm{T}_{H} 1\right.$ cell). There are two main subsets of activated CD 4

$T$ cells: $T_{H} 1$ cells and $T_{H} 2$ cells. $T_{H} 1$ cells produce interferon- $\gamma$ and tumour-necrosis factor, thereby promoting cellmediated immunity. $T_{H} 2$ cells produce interleukin-4 (IL-4), IL-5 and IL-13, thereby supporting humoral immunity and counteracting $\mathrm{T}_{\mathrm{H}} 1$-cell responses.

$\mathrm{CD} 4+\mathrm{CD} 25+$ regulatory $\mathrm{T}$ cell

$\left(T_{\text {Reg }}\right.$ cell). A T cell that expresses CD4, CD25 also

known as the interleukin-2 receptor $\alpha$-chain) and the transcription factor T-bet, and is activated in an antigenspecific, MHC-class-II-restricted manner, but inhibits T cells and natural killer cells in a nonspecific manner, in part by producing transforming growth factor $\beta$.

Chediak-Higashi syndrome A rare autosomal recessive genetic disorder that is caused by mutations in the gene CHS I (Chediak-Higashi syndrome 1; also known as LYST), which is involved in lysosome fission and secretion.

Oncogene

A gene that when overexpressed or when incorporating a gain-of-function mutation contributes to oncogenesis.

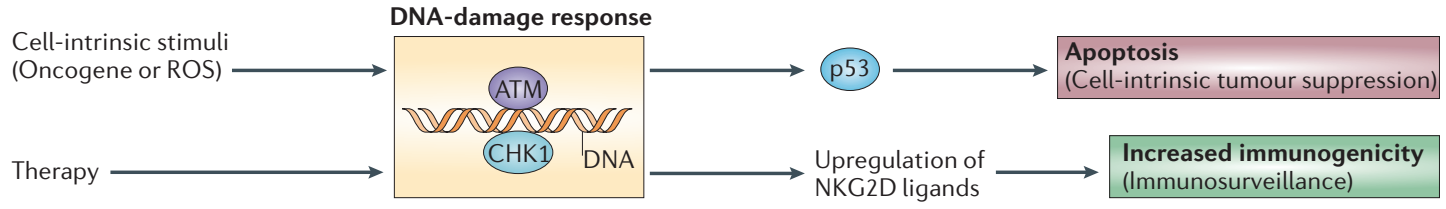

Figure 3 | Hypothetical links between endogenous tumour suppression and immunosurveillance. Activation of the DNA-damage response by cell-intrinsic stimuli (oncogene activation or reactive oxygen species, ROS) or therapy activates the ATM (ataxia-telangiectasia mutated)-CHK1 (checkpoint kinase 1 homologue) pathway. This pathway leads either to $\mathrm{p} 53$-dependent apoptosis of tumour cells or to increased immunogenicity of tumour cells as a result of upregulation of NKG2D (natural-killer group 2, member D) ligands, which increases recognition of tumour cells by NKG2D-expressing NK cells, natural killer T (NKT) cells and T cells.

regulatory $T\left(T_{\text {Reg }}\right)$ cells in the tumour is a predictor of reduced patient survival ${ }^{21,22}$. Accordingly, ex vivo depletion of $\mathrm{T}_{\mathrm{Reg}}$ cells from TIL populations recovered from renal-cell carcinomas or melanomas increases the lysis of tumour cells by NK cells ${ }^{23}$. Similarly, the responses of TILs to prostate-cancer cells can be restored ex vivo by inhibiting two immunosuppressive enzymes that are produced by tumour cells: arginase- 1 and nitric-oxide synthase 2 (NOS2; also known as iNOS) ${ }^{24}$. These findings indicate that, although human tumours are often infiltrated by TILs, the antitumour activity of these T cells seems to be inhibited.

Additional evidence that supports the idea that immunosurveillance has a role in the suppression of human cancer is provided by the finding that immunodeficiencies predispose patients to the development of cancer. Immunosuppressed transplant recipients have an increased risk of developing certain cancers, including cancers without a known viral aetiology, such as melanoma ${ }^{25,26}$. However, such patients have no increased risk of developing gastrointestinal, urogenital or respiratory cancers $^{25}$, indicating that the mere suppression of the immune system by glucocorticoids and cyclosporin A (both of which mainly affect T cells) is not sufficient to increase the incidence of such cancers. Patients with Chediak-Higashi syndrome, an autosomal recessive disorder that is characterized by abnormal cytotoxic function of NK cells, have a 200 -fold higher risk of developing malignancy than do individuals who do not suffer from this syndrome ${ }^{27}$. In addition, biallelic mutations of the gene that encodes perforin might predispose patients to the development of Hodgkin's lymphoma or non-Hodgkin's lymphoma ${ }^{28}$. Similarly, a polymorphism in CD95 ligand (CD95L; also known as FASL) predisposes patients to increased activation-induced cell death of $\mathrm{T}$ cells and to cervical cancer ${ }^{29}$. Carrying specific combinations of HLA alleles and alleles that encode the cognate killer-cell immunoglobulin-like receptors (KIRs), which are expressed by NK cells, also results in a predisposition to cervical cancer ${ }^{30}$. Moreover, the presence of certain human NKG2D alleles, which affect the natural cytotoxic activity of peripheral-blood lymphocytes, is associated with a risk of developing cancer at any site ${ }^{31,32}$. For example, two common NKG2D haplotypes (the low NK-cell-activity-related LNK1/ LNK2 haplotype, and the high NK-cell-activity-related HNK1/HNK2 haplotype) correlated with the degree of spontaneous lysis of the tumour-cell line K-562, which is an NKG2D-dependent target ${ }^{32}$. The HNK1/HNK1 haplotype was associated with a lower risk of cancer than the LNK1/LNK1 haplotype ${ }^{33}$.

Together, the data from human studies support the existence of a cancer-immunosurveillance system that involves $\mathrm{CD}^{+} \mathrm{T}$ cells, $\mathrm{T}_{\mathrm{H}} 1$ cells and NK cells and is locally suppressed by $\mathrm{T}_{\mathrm{Reg}}$ cells and tumour-cell products. Moreover, data from several studies ${ }^{31-33}$ support the concept that a two-hit process facilitates the development of cancer: the first hit being the cell-intrinsic oncogenic event, and the second being an innate or adaptive deficiency in immune recognition of, or effector function against, tumour cells. Nevertheless, whether immunosurveillance and its avoidance have a role in all types of human cancer or whether they influence only a few types requires further investigation.

\section{Intrinsic and extrinsic barriers to carcinogenesis}

There are numerous tumour-cell-intrinsic barriers that inhibit the development of cancer and that lead to the suppression of tumours ${ }^{34}$. For example, oncogene-driven tumour-cell division can trigger DNA damage associated with DNA replication, which then activates the DNA-damage response. This response involves the proteins ataxia-telangiectasia mutated (ATM), checkpoint kinase 1 homologue (CHK1) and p53, and it leads to cell-cycle arrest or apoptosis ${ }^{35,36}$. Therefore, evidence consistent with activation of the DNA-damage response, as well as increased apoptosis, is frequently observed in early superficial lesions (for example, in pre-malignant lesions of bronchi, intraductal breast carcinomas and dysplastic nevi), yet these processes do not occur after full development of carcinoma or melanoma, owing to inactivation of the ATM-CHK1-p53 pathway ${ }^{35,36}$. Intriguingly, the DNA-damage response can also activate the expression of ligands for NKG2D - such as MHC-class-I-polypeptide-related sequence A (MICA) and retinoic acid early transcript 1 (RAE1) - by tumour cells, rendering the tumour cells immunogenic and possibly susceptible to killing by NKG2D-expressing cells, such as NK cells, NKT cells, $\gamma \delta$ T cells and some cytolytic CD8 ${ }^{+} \alpha \beta$ T cells. The upregulation of expression of such NKG2D ligands involves the activation of ATM, ATR (ATM and Rad3 related) and CHK1 (REF. 37), indicating that similar or overlapping pathways couple chronic activation of the DNA-damage response to 
cell-intrinsic and cell-extrinsic (immune-mediated) pathways of suppression of tumours (FIG. 3).

With regard to cell-extrinsic mechanisms of tumour suppression, neutralization of NKG2D by injection of a specific antibody increases the sensitivity of mice to the development of methylcholanthrene (MCA)induced fibrosarcoma ${ }^{38}$. MCA-induced fibrosarcomas from perforin-deficient mice more frequently express the NKG2D ligand RAE1 than do fibrosarcomas from perforin-sufficient mice. Moreover, the fibrosarcomas that arise in perforin-deficient mice are immunogenic when transferred to wild-type syngeneic mice ${ }^{38}$, consistent with a role for perforin (and NKG2D) in the selection of non-immunogenic tumour-cell variants (that is, in immunoselection). In addition, DNA-based vaccines that encode syngeneic or allogeneic NKG2D ligands plus tumour antigens (such as survivin and carcinoembryonic antigen) elicit both NK-cell-mediated and $\mathrm{CD}^{+} \mathrm{T}$-cell-mediated anticancer immune responses ${ }^{39}$, indicating that the recognition of NKG2D ligands might have a co-stimulatory effect on T cells. Human tumour cells often overexpress the NKG2D ligands MICA and MICB, and cell-surface expression of MICA is an indicator of good prognosis in patients with colorectal carcinoma ${ }^{40}$. Conversely, proteolytic shedding of MICA and MICB from tumour cells, as occurs in advanced human cancers ${ }^{41}$, might contribute to immunosubversion by ligating NKG2D and reducing its expression at the cell surface of lymphocytes, thereby inhibiting lymphocyte function. This might occur because soluble (in contrast to membrane-bound) NKG2D ligands cause internalization of NKG2D ${ }^{41}$. Constitutive expression of the mouse NKG2D ligand RAE1 (encoded by a transgene in normal epithelial cells), or transplantation of mice with RAE $1^{+}$tumours, causes downregulation of NKG2D expression by CD8 ${ }^{+} \mathrm{T}$ cells and NK cells (both those that are infiltrating the tumour and those present in the blood), and this effect was found to be coupled to a generalized defect in NK-cell-mediated cytotoxicity ${ }^{42}$. Importantly, Rae1e-transgenic mice with an inactive NKG2D system are more susceptible to chemical-induced carcinogenesis than are wild-type mice ${ }^{42}$, underlining the role of NKG2D in immuno-surveillance at early stages of tumour development.

Although there seems to be a link between upstream events in the DNA-damage response (those involving ATM, ATR and CHK1) and expression of NKG2D ligands ${ }^{37}$, there are no data that establish such a link for the downstream effector of the DNAdamage response, p53. Nevertheless, in addition to the induction of apoptosis, p53 has been implicated in providing a cell-intrinsic barrier to the development of cancer by mediating cellular senescence: that is, by inducing a permanent arrest in the G0/1 (gap 0/1) phase of the cell cycle ${ }^{43,44}$. The impact of cancer-cell senescence on the immune response is unknown, although it has been inferred that senescent tumour cells might 'hide' from the immune system ${ }^{45}$. Indeed, one of the markers of senescent tumour cells, decoy receptor 2 (also known as TNFRSF10D), suppresses TRAIL-induced apoptosis ${ }^{46}$. Interestingly, it seems that type I IFNs (that is, IFN $\alpha$ and IFN $\beta$ ), which have an important role in immunosurveillance ${ }^{47}$, can upregulate the p53-mediated response of tumour cells ${ }^{48}$. Therefore, MCA-induced fibrosarcomas derived from IFN $\alpha$-receptor-1-deficient mice are rejected in a lymphocyte-dependent manner when transplanted to wild-type mice. However, recent studies indicate that tumour cells (and by extension p53) are not important targets of endogenously produced type I IFNs. Instead, type I IFNs mediate their antitumour effects through host haematopoietic cells ${ }^{47}$. So the role of crosstalk between type I IFNs and p53 in mediating tumour suppression at the intersection between cell-intrinsic and cell-extrinsic (immunemediated) pathways remains to be established. It is possible, however, that loss of p53 function, which is a frequent occurrence in developing cancers, might affect the recognition of tumour cells by the immune system. For example, for human keratinocytes, loss of p53 increases susceptibility to type-I-IFN-induced, TRAIL-dependent apoptosis ${ }^{49}$. Whether such a mechanism contributes to the increased susceptibility of cancers to TRAIL-induced apoptosis remains to be established.

Taken together, the evidence indicates that cellintrinsic responses that participate in the suppression of tumours are linked to the expression of immunostimulatory NKG2D ligands and, perhaps, to the modulation of TRAIL-dependent apoptosis.

\section{The six cell-intrinsic hallmarks of cancer}

There are numerous molecular differences between cancer cells and healthy cells. These differences can be classified into six characteristic changes ${ }^{1}$. Here, we briefly discuss the links between these cancer-specific characteristics and immunosurveillance (TABLE 1).

Self-sufficiency in growth signals. Although most soluble mitogenic growth factors are produced by one cell type to stimulate proliferation of another (a process known as heterotypic signalling), many cancer cells acquire the ability to synthesize growth factors to which they are responsive. This creates a positivefeedback signalling loop that is known as autocrine stimulation. Some of the autocrine growth factors that are produced by tumour cells not only provide growthstimulatory signals but also subvert the immune response simultaneously (TABLE 1). For example, IL-4 and IL-10 are autocrine growth factors for thyroid carcinoma ${ }^{50}$, and both of these cytokines can polarize the $\mathrm{T}$-cell response from a $\mathrm{T}_{\mathrm{H}} 1$-cell response to a $\mathrm{T}_{\mathrm{H}} 2$ cell response, thereby inactivating anticancer immunity, which often relies on $\mathrm{T}_{\mathrm{H}} 1$-cell responses. IL- 6 is one of the most important autocrine growth factors in the pathogenesis of numerous cancers, such as prostate cancer, renal cancer and myeloma. Aberrant activation of STAT3, which is involved in signalling through the IL- 6 receptor, has been shown to inhibit inflammatory responses and crosstalk between innate and adaptive immune responses in various human cancers, thereby favouring unrestrained tumour growth ${ }^{51}$. STAT3 can be activated by other growth 
factors, particularly by the oncogenic fusion protein of PAX3 (paired box protein 3) and FKHR (forkhead homologue 1, rhabdomyosarcoma; also known as FOXO1A $)^{52}$, which is associated with the alveolar subtype of rhabdomyosarcoma. So PAX3-FKHR has a dual function: it favours the transformation of the cell while causing reduced expression of $\mathrm{MHC}$ molecules and increased production of IL-10 by tumour cells, to inhibit surrounding inflammatory cells and detection by the immune system ${ }^{52}$. IL- 6 and IL- 10 have also been found to stimulate a B7-H4-expressing T-cellsuppressive macrophage population in patients with ovarian carcinoma ${ }^{53}$.

Overexpression of growth-factor receptors might enable a cancer cell to become hyper-responsive to ambient amounts of growth factors that would not normally trigger proliferation, or such overexpression might cause ligand-independent signalling.
For example, the epidermal-growth-factor receptor (EGFR; also known as ERBB1) is overexpressed in stomach, brain and breast tumours, whereas expression of the receptor ERBB2 is upregulated in stomach and breast carcinomas. Both EGFR and ERBB2 are important tumour antigens for the induction of T-cell responses, and patients with tumours that overexpress either of these growth-factor receptors often mount immune responses to EGFR- or ERBB2-derived peptides $^{54}$. Another way in which growth signals can be provided is an activating mutation of signal transducers such as Ki-RAS, which is frequently mutated in colon carcinoma. Mutated Ki-RAS has been assessed as a potential antigen for incorporation in antitumour vaccines, and this approach is yielding promising clinical data ${ }^{55}$. However, to our knowledge, no cases of spontaneous immune responses to Ki-RAS have been reported in patients with cancer.

\begin{tabular}{|c|c|c|c|}
\hline Cancer hallmark & $\begin{array}{l}\text { Factor involved or } \\
\text { characteristic }\end{array}$ & Effect on immune response & Refs \\
\hline \multirow{3}{*}{$\begin{array}{l}\text { Self-sufficiency in } \\
\text { growth signals }\end{array}$} & $\mathrm{IL}-4$ & - Promotes differentiation into $\mathrm{T}_{\mathrm{H}} 2$ cells & 50 \\
\hline & IL-6 & $\begin{array}{l}\text { - Inhibits inflammatory response, through } \\
\text { aberrant JAK-STAT3 signalling }\end{array}$ & 51,53 \\
\hline & IL-10 & - Promotes differentiation into $\mathrm{T}_{\mathrm{H}} 2$ cells & $50,52,53$ \\
\hline $\begin{array}{l}\text { Insensitivity to } \\
\text { antigrowth signals }\end{array}$ & TGF $\beta$ resistance & $\begin{array}{l}\text { - Inhibits antigen presentation } \\
\text { - Inhibits T-cell proliferation } \\
\text { - Inhibits NK-cell cytotoxicity } \\
\text { - Activates } \mathrm{T}_{\text {Reg }} \text { cells }\end{array}$ & $23,57,58$ \\
\hline \multirow[t]{4}{*}{ Evasion of apoptosis } & BCL-2-family proteins & - Tumour antigens & 66 \\
\hline & MUC1 & $\begin{array}{l}\text { - Subverts DC function } \\
\text { - Tumour antigen }\end{array}$ & 68,69 \\
\hline & Survivin & $\begin{array}{l}\text { - Promotes T-cell apoptosis, through inducing } \\
\text { CD95L expression by tumour cells } \\
\text { - Tumour antigen }\end{array}$ & 71,72 \\
\hline & Reduced caspase activation & - Reduces tumour immunogenicity & 82 \\
\hline \multirow{2}{*}{$\begin{array}{l}\text { Limitless replicative } \\
\text { potential }\end{array}$} & Mutated p53 & - Tumour antigen & 86 \\
\hline & TERT & - Tumour antigen & 84,85 \\
\hline \multirow[t]{2}{*}{ Sustained angiogenesis } & $\operatorname{cox} 2$ & $\begin{array}{l}\text { - Promotes } T_{H} 2 \text {-cell responses } \\
\text { - Inhibits T-cell cytotoxicity }\end{array}$ & $90-92$ \\
\hline & VEGF & $\begin{array}{l}\text { - Inhibits T-cell activation } \\
\text { - Inhibits NF- } \mathrm{BB} \text { activation in DCs }\end{array}$ & 87,88 \\
\hline \multirow[t]{3}{*}{$\begin{array}{l}\text { Tissue invasion and } \\
\text { metastasis }\end{array}$} & Reduced NECL2 expression & $\begin{array}{l}\text { - Inhibits NK-cell cytotoxicity, through CRTAM } \\
\text { - Inhibits CD8 } 8^{+} \text {-cell IFN } \gamma \text { production, through } \\
\text { CRTAM } \\
\text { - Leads to loss of adherence at epithelial cell-cell } \\
\text { junctions }\end{array}$ & 93 \\
\hline & Altered lysosome trafficking & $\begin{array}{l}\text { - Increases immunogenicity, through increasing } \\
\text { cell-surface HSP70 }\end{array}$ & 95,96 \\
\hline & MMPs & $\begin{array}{l}\text { - Induce cleavage of CD25 } \\
\text { - Activate TGF } \beta \\
\text { - Promote shedding of ICAM1 and ICAM2 }\end{array}$ & 98 \\
\hline
\end{tabular}

BCL-2, B-cell lymphoma 2; CD95L, CD95 ligand; COX2, cyclooxygenase-2; CRTAM, MHC-class-I-restricted T-cell-associated molecule; DC, dendritic cell; HSP70, heat-shock protein 70; ICAM, intercellular adhesion molecule; IFN $\gamma$, interferon- $\gamma$; IL, interleukin; JAK, Janus kinase; MMP, matrix metalloproteinase; MUC1, mucin-1; NECL2, nectin-like 2; NF-KB, nuclear factor-KB; NK cell, natural killer cell; p53, tumour-suppressor protein p53; STAT3, signal transducer and activator of transcription 3; TERT, telomerase reverse transcriptase; TGF $\beta$, transforming growth factor- $\beta ; \mathrm{T}_{\mathrm{H}} 2$ cell, T helper 2 cell; $\mathrm{T}_{\text {Req }}$ cell, $C D 4^{+} \mathrm{CD} 25^{+}$regulatory $\mathrm{T}$ cell; $\mathrm{VEGF}$, vascular endothelial growth factor. 
These findings indicate that tumour cells can produce soluble factors that affect the tumour and the immune system of the host, and that activating mutations in growth-stimulatory signal transducers can alter the antigenic profile of tumour cells.

Mitochondrial outermembrane permeabilization (MOMP). An apoptosisassociated process that results in the release of apoptosisinducing proteins - such as cytochrome $c$, apoptosisinducing factor, and second mitochondria-derived activator of caspase (also known as DIABLO) - from the mitochondrial intermembrane space into the cytosol.

\section{Death receptors}

A family of cell-surface receptors that can mediate cell death following ligand-induced trimerization. The best-studied members include tumournecrosis-factor (TNF) receptor 1, CD95 (also known as FAS) and two receptors for TNF-related apoptosisinducing ligand (TRAILR 1 and TRAILR2).

\section{Caspases}

A family of cysteine proteases that cleave proteins carboxyterminal to asparagine residues. Initiator caspases are typically activated in response to particular stimuli: for example, caspase- 8 is activated after death-receptor ligation; caspase-9, after apoptosome activation; caspase-2, after DNA damage. By contrast, effector caspases (that is, caspase-3, caspase-6 and caspase-7) are particularly important for the ordered dismantling of vital cellular structures.

B-cell-lymphoma-2 family (BCL-2 family). A family of proteins that contain at least one BCL-2-homology domain $(\mathrm{BH})$. The family is classified into three groups: antiapoptotic multidomain proteins, such as BCL-2, BCL-X and MCL1 (myeloid-cell leukaemia sequence 1), which contain four BHs; pro-apoptotic multidomain proteins, such as BAX (BCL-2associated $X$ protein) and $B A K$ (BCL-2 antagonist/killer), which contain three $\mathrm{BHs}$; and a pro apoptotic subfamily of proteins that contain only one $\mathrm{BH}$, the $\mathrm{BH} 3$-only proteins.
Insensitivity to antigrowth signals. In a normal tissue, multiple antiproliferative signals operate to maintain cell quiescence and tissue homeostasis. Such signals include those mediated by both soluble growth inhibitors and immobilized growth inhibitors that are embedded in the extracellular matrix and on the surface of neighbouring cells. One of the most important antiproliferative signals is mediated by transforming growth factor- $\beta$ (TGF $\beta$ ), and many tumours disable components of the TGF $\beta$ mediated signalling pathway (TABLE 1): for example, by mutation or loss of TGF $\beta$ receptors, by mutation of the transcription factor SMAD4 (mothers against decapentaplegic homologue 4), or by deletion of the loci that encode INK4B (also known as p15) and retinoblastoma protein. Moreover, TGF $\beta$ favours the epithelial to mesenchymal transition of established breast-cancer cell lines and therefore might function as an autocrine and paracrine factor that allows tumour-cell motility, invasiveness and metastasis ${ }^{56}$. In addition, TGF $\beta$ is one of the most potent known immunosuppressive agents and functions at several levels. Among other functions, TGF $\beta$ reduces the amount of antigen presentation by DCs, inhibits the activity of IFN $\gamma$, reduces the proliferation of T cells, suppresses the cytotoxic activity of NK cells and stimulates the proliferation of $\mathrm{T}_{\mathrm{Reg}}$ cells ${ }^{57}$. Recent data also indicate that a specific subset of tumour-associated DCs can stimulate the proliferation of $\mathrm{T}_{\text {Reg }}$ cells in a TGF $\beta$ dependent manner ${ }^{58}$ and that $\mathrm{T}_{\mathrm{Reg}}$ cells that accumulate in the tumour bed express membrane-bound TGF $\beta^{23}$. By contrast, it is thought that TGF $\beta$ can also have immunostimulatory effects (in particular, on specific $\mathrm{T}_{\mathrm{H}}$-cell subsets ${ }^{59}$ ), so it would be simplistic to suggest that TGF $\beta$ is always immunosuppressive. However, there are data that point to the in vivo relevance of endogenous TGF $\beta$ : adoptive transfer of TGF $\beta$-desensitized CD8 ${ }^{+}$ $\mathrm{T}$ cells that had been transfected with a dominantnegative TGF $\beta$ receptor 2 was found to be particularly efficient at eradicating prostate cancer in mice $e^{60}$.

Taken together, these data highlight that, by ignoring TGF $\beta$-mediated growth-inhibitory signals, tumour cells can replicate in an immunosuppressive environment that is rich in TGF $\beta$.

Evasion of apoptosis. The apoptotic programme is carried out through two main pathways: the mitochondrial pathway, which involves mitochondrial outer-membrane permeabilization (MOMP) ${ }^{61}$; and the death-receptor pathway, which involves ligation of a plasma-membrane receptor, leading to the formation of a death-inducing signalling complex (DISC) ${ }^{62}$. Both pathways culminate in the activation of caspases and caspase-independent cell-death pathways ${ }^{63}$. Defective apoptosis is not crucial for initial oncogenesis, but it contributes to the acquisition of resistance to chemotherapy and to immune effectors $^{64}$. Tumour cells can develop resistance to the main apoptosis-inducing effector molecules of the innate and adaptive immune systems (TABLE 1). For example, aggressive tumour variants that are selected in vivo in mice that have been administered tumour-antigenspecific cytotoxic T lymphocytes (CTLs) are particularly resistant to IFN $\gamma$-induced transcriptional effects, including IFN $\gamma$-induced expression of CD95 and killing induced by CD95 (REF. 65).

Important endogenous inhibitors of MOMP are the anti-apoptotic proteins of the B-cell lymphoma 2 (BCL-2) family, which includes BCL-2, BCL- $\mathrm{X}_{\mathrm{L}}$ and myeloid-cell leukaemia sequence 1 (MCL1). It has been reported that patients with cancer at various sites have peripheral-blood CTLs that recognize peptides derived from these proteins, whereas healthy individuals lack such spontaneous immunity to BCL-2, BCL- $\mathrm{X}_{\mathrm{L}}$ and MCL1 (REF. 66). Other relevant inhibitors of MOMP that do not belong to the BCL-2 family have recently been identified. The carboxyterminal subunit of the epithelial-cell protein MUC1 localizes to mitochondria and blocks stress-induced activation of the mitochondrial apoptotic pathway ${ }^{67}$. MUC1 - which is a heterodimeric transmembrane glycoprotein that is overexpressed by most human carcinomas - is a T-cell antigen that is presented by various tumours, such as breast, colon, pancreatic, ovarian and lung carcinomas. Owing to its differential glycosylation in normal cells and tumour cells, MUC1 might be tumour-cell-specific antigen. In addition, MUC1 can have several immunosuppressive effects: inhibiting the differentiation of monocytes into DCs, skewing the differentiation of DCs into cells with an IL- $10^{\text {hi }} \mathrm{IL}-12^{\text {low }}$ regulatory phenotype ${ }^{68}$, and functioning as a chemoattractant for immature DCs and then subverting their ability to stimulate $\mathrm{T}_{\mathrm{H}} 1$-cell responses ${ }^{69}$. Another inhibitor of MOMP that is relevant to cancer is survivin ${ }^{70}$, which is also an important tumour antigen $^{71}$. For example, survivin induces upregulation of expression of the death-receptor ligand CD95L by colon carcinomas $^{72}$. The expression of CD95L at the surface of tumour cells might contribute to the deletion of CD95expressing activated $\mathrm{T}$ cells that invade the tumour ${ }^{73}$, although this possibility is controversial. CD95L might also elicit an inflammatory respons $\mathrm{e}^{74}$. Irrespective of whether survivin overexpression or other mechanisms account for the expression of CD95L by tumours, this expression is associated with a poor prognosis for patients with various conditions, such as Barrett's oesophagus ${ }^{75}$ and colon carcinoma ${ }^{76}$, for which CD95L expression has been associated with increased apoptosis of T cells.

Tumour cells often lose the expression of functional APAF1 (apoptotic-protease-activating factor 1), which is required for the apoptosome-dependent activation of caspases after MOMP. Moreover, tumours often overexpress inhibitor-of-apoptosis proteins (IAPs) - such as XIAP (X-linked IAP) and IAP1 - which inhibit caspases. Although the inactivation of APAF1 (REF. 77) or the presence of IAPs is likely to have little impact on the survival of tumour cells (which remain susceptible to caspase-independent cell death) ${ }^{63}$, these factors are associated with a poor prognosis for patients with head and neck squamous-cell carcinoma (IAP1 overexpression) ${ }^{78}$, acute de novo myeloid leukaemia (XIAP overexpression) ${ }^{79}$, 


\section{Box 1 | Immunogenic versus non-immunogenic cell death}

Despite a growing body of research, whether an immune response is triggered in response to dying tumour cells remains an open question ${ }^{18}$. In particular, the precise phenomenological and mechanistic relationship between the different modalities of cell death and the immunogenicity of cell death is unknown. Most of the studies that have been carried out so far have concentrated on the dichotomy between apoptosis and necrosis, but such studies have not addressed the issue of the biochemistry of dying cells.

It has long been thought that apoptotic cell death is poorly immunogenic (or tolerogenic), whereas necrotic cell death is immunogenic. This difference was thought to result from an intrinsic capacity of cells dying from non-apoptotic cell death to stimulate the immune response: for example, by stimulating local inflammatory responses (in response to 'danger' signals) and/or by triggering the maturation of dendritic cells.

It seems, however, that the classification of necrosis as immunogenic and apoptosis as tolerogenic is an oversimplification. It has recently been shown that the capacity of apoptotic cells to trigger an immune response depends on the inducer of apoptosis ${ }^{82}$, indicating that qualitative differences in the biochemical mechanisms of cellular apoptosis could lead to qualitative differences in the resultant immune response. For example, anthracycline-treated tumour cells were found to be particularly immunogenic ${ }^{82}$, whereas mitomycin-C-treated tumour cells were not immunogenic. Therefore, future studies will need to correlate antitumour immune responses with biochemically (rather than morphologically) defined cell-death modalities, as well as with spatiotemporal cell-death patterns, both in animals and in patients. Indeed, the induction of immunogenic cell death of cancer cells should be one of the aims of anticancer chemotherapy, because the immune system can then contribute, through a 'bystander' effect, to eradicate chemotherapy-resistant cancer cells and cancer-cell precursors.

or melanoma or colorectal carcinoma (reduced APAF1 expression $)^{80,81}$. Nevertheless, it is possible that the lack of caspase activation might affect the immunogenicity of cell death (BOX 1). Indeed, activation of caspases might be required to elicit immunogenic tumour-cell death in an in vivo model of anthracycline-mediated antitumour chemotherapy ${ }^{82}$, and an adenovirus that encodes caspase- 1 and IL-12 was found to improve the survival of mice with adenocarcinoma of the prostate ${ }^{83}$.

Evolving pre-malignant and malignant cell populations show chronic, widespread apoptosis and therefore suffer considerable cell attrition concomitant with cell accumulation. As a result, reducing the immunogenicity of apoptosis might be an important strategy that tumours use to avoid destruction by the immune system, not only after episodes of apoptosis-inducing chemotherapy but also during spontaneous cancer growth.

Limitless replicative potential. Replication without limits requires maintenance of the ends of the chromosomes, which are known as telomeres. Most (85-90\%) malignant cells succeed in doing so by upregulating expression of the enzyme telomerase, which adds hexanucleotide repeats to the ends of telomeric DNA. The remaining $10-15 \%$ of malignant cells can activate a mechanism that is known as alternative lengthening of telomeres, which seems to maintain telomeres through recombination-based interchromosomal exchanges of sequence information. The catalytic subunit of human telomerase, TERT (telomerase reverse transcriptase), is a tumour antigen to which patients with malignant disease, such as breast cancer ${ }^{84}$ and chronic myeloid leukaemia ${ }^{85}$, mount immune responses. In addition, to ensure replicative potential, tumour cells can have mutations in, or lose expression of, senescence-inducing proteins, such as p53 (which can also function as a tumour antigen $)^{86}$ (TABLE 1). In tumours that do not express p53, expression of the cell-cycle regulator cyclin B1 is increased, and cyclin B1 is also a potential tumour antigen ${ }^{15}$. However, there are no known immunomodulatory effects of cancer-cell immortalization, apart from the previously mentioned tumour-specific epitopes that can elicit spontaneous immune responses.

Sustained angiogenesis. Many tumour cells show higher expression of vascular endothelial growth factor (VEGF) and/or fibroblast growth factor 1 (FGF1) and FGF2 than their counterparts in normal tissue, and these factors are crucial for mediating an 'angiogenic switch' from the state of vascular quiescence. In addition to promoting angiogenesis, VEGF might inhibit antitumour immunity, both by inhibiting the activation of nuclear factor- $\kappa \mathrm{B}$ in DCs, thereby preventing DC maturation ${ }^{87}$, and by suppressing the activation of $\mathrm{T}$ cells ${ }^{88}$ (TABLE 1). Consistent with these postulated immunosuppressive functions of VEGF, administration of VEGF-specific antibody increases the efficacy of immunotherapy in mouse models ${ }^{89}$.

Overexpression of the pro-inflammatory mediator cyclooxygenase-2 (COX2) is a common characteristic of various pre-malignant and malignant lesions of epithelial-cell origin in organs such as the colon, lungs, breasts, prostate, bladder, stomach and oesophagus. Moreover, inhibition of COX2 has been shown to have chemopreventive and, perhaps, chemotherapeutic effects in various human cancers, particularly in colon cancer. COX2 causes the local overproduction of prostaglandin $\mathrm{E}_{2}\left(\mathrm{PGE}_{2}\right)$, which is thought to have an important role in favouring angiogenesis, partly through the induction of VEGF production ${ }^{90}$. Moreover, COX2 and prostanoids, particularly $\mathrm{PGE}_{2}$, suppress antitumour immunity, by suppressing macrophage-mediated or T-cell-mediated tumour killing and by polarizing the balance of $\mathrm{T}_{\mathrm{H}}$-cell responses towards $\mathrm{T}_{\mathrm{H}} 2$-cell responses ${ }^{90}$. Accordingly, selective inhibition of COX2 can restore the tumour-induced imbalance between IL-10 ( $\mathrm{a} \mathrm{T}_{\mathrm{H}} 2$ cytokine) and IL-12 (a $\mathrm{T}_{\mathrm{H}} 1$ cytokine) in mice with lung cancer ${ }^{91}$ and can re-establish impaired mononuclear-cell function in patients with head and neck cancer ${ }^{92}$.

Tissue invasion and metastasis. The settling of tumour cells in locations that are distant from the primary tumour - metastasis - is the cause of $90 \%$ of human deaths from cancer, and selective pressure is likely to guide cancer cells to niches where they can 'hide' from the immune response. The invasion of distant sites by tumour cells requires alterations in the proteins that are involved in the tethering of cells to their surroundings, as well as in the secretion of proteases. In terms of tethering, the tumour-suppressor gene TSLC1 (tumour suppressor in lung cancer 1), which is frequently inactivated in nonsmall-cell lung-cancer cells, encodes the protein nectinlike 2 (NECL2). NECL2 mediates epithelial-cell junctions though homotypic and/or heterotypic interactions with other nectins or nectin-like proteins. Moreover, NECL2 is recognized by CRTAM (MHC-class-I-restricted T-cell-associated molecule), which is expressed by NK cells and $\mathrm{CD}^{+} \mathrm{T}$ cells, and promotes the cytotoxicity of 


\section{Cathepsins}

A class of cysteine proteases that are localized mainly in lysosomes and lysosome-like organelles.

Damage-associated molecular pattern molecules

(DAMPs). As a result of cellular stress, cellular damage and non-physiological cell death, DAMPs are released from the degraded stroma (for example, hyaluronate), from the nucleus (for example, high-mobility group box 1 protein, HMGB 1) and from the cytosol (for example, ATP, uric acid, S100 calcium-binding proteins and heat-shock proteins). Such DAMPs are thought to elicit local inflammatory reactions.

Cross-priming

The initiation of a CD8 + T-cell response to an antigen that is not present within antigenpresenting cells (APCs). This occurs through the ability of certain APCs to present peptides that are derived from exogenous antigens in the context of MHC class I molecules. This property is atypical, because the peptides that are presented in the context of $\mathrm{MHC}$ class molecules by most cells are derived from endogenous proteins.

Exosomes

Small lipid-bilayer vesicles that are released from dendritic cells (DCs) and tumour cells. Characteristically, exosomes are enriched in $\mathrm{MHC}$

complexes, tetraspanins, and heat-shock protein 70 (HSP70) and HSP90, all of which could be cross-presented by DCs to activate T cells.
NK cells and production of IFN $\gamma$ by T cells ${ }^{93}$. Therefore, inactivation of NECL2 expression has a dual oncogenic effect: it allows the disorganization of epithelial-cell layers, and it removes a protein that is recognized by NK cells and T cells (TABLE 1).

The secretion of proteolytic enzymes, such as cathepsins, is also involved in the local invasion of a tissue by tumour cells. One of the hallmarks of cancer is increased secretion of lysosome-resident proteases, a process that seems to be associated with increased amounts of heat-shock protein 70 (HSP70) in both the lysosome membrane and the plasma membrane $e^{94}$. In theory, this could increase the immunogenicity of tumour cells, because HSP70 is thought to be one of the main damageassociated molecular pattern molecules and can chaperone tumour peptides for the cross-priming of $\mathrm{CD}^{+} \mathrm{T}$ cells $s^{95,96}$. Moreover, HSP70 ${ }^{\text {hi }}$ tumour cells are killed more efficiently by NK cells than are their HSP70 ${ }^{\text {low }}$ counterparts. Importantly, colon- and pancreatic-carcinoma cells can release HSP70-bearing exosomes that stimulate the cytolytic activity of NK cells against tumours ${ }^{97}$.

Local invasion is also mediated by matrix metalloproteinases (MMPs) that are secreted by tumour cells and stromal cells. MMPs are also involved in the escape of cancer cells from immunosurveillance: for example, through MMP9-mediated cleavage of CD25, activation of TGF $\beta$, and shedding of intercellular adhesion molecule 1 (ICAM1) and ICAM2 from tumour cells ${ }^{98}$.

These examples illustrate that the molecular features that determine the capacity of tumour cells to invade tissues and to metastasize also affect the immunogenicity of tumour cells.

\section{The seventh hallmark of cancer}

In humans, tumours develop a series of strategies to evade immunosurveillance, and these strategies are presumably unrelated to the other characteristics of carcinogenesis and result from the selective pressure exerted by the immune system. These strategies are known as immunoselection. One common strategy to elude a T-cell-mediated immune response is the downregulation or loss of expr ession of HLA class I molecules, as has been documented for a large range of epithelial-cell cancers and melanomas. Loss of HLA class I expression is common in lung cancer, and it has been postulated that only immunoselected tumour cells that lack HLA class I expression can escape immune attack and develop into cancer ${ }^{99}$. Similarly, tumours often downmodulate molecules that are involved in antigen processing and in antigen presentation by HLA class I molecules including transporter associated with antigen processing 1 (TAP1), low-molecular-mass protein 2 (LMP2), LMP7 and tapasin - the expression of which is progressively lost during the development of colorectal carcinoma ${ }^{100}$ (FIG. 4a). Tumour cells can also develop mechanisms to avoid being killed by CTLs. Overexpression of the serine-protease inhibitor PI9 by tumour cells efficiently blocks the granzyme-B-perforin pathway of target-cell lysis ${ }^{101}$. Similarly, additional outcomes of immunoselection include downregulation or mutation of death receptors, methylation or mutation of the gene that encodes caspase-8, and overexpression of FLIP (caspase-8 (FLICE)-like inhibitory protein) or decoy receptors for TRAIL, all of which cause resistance to CTL-induced killing of tumour cells ${ }^{102}$.

Several tumour products that are dispensable for cellintrinsic cancer-cell characteristics (discussed earlier) might be involved in immunosubversion: that is, the active suppression of the immune response. For example, some tumours (or tumour-associated myeloid cells) overproduce nitric oxide and have increased arginase-1 activity, both of which can inhibit T-cell function ${ }^{103}$ (FIG. 4b). More importantly, tryptophan degradation by indoleamine 2,3-dioxygenase (IDO), which is constitutively expressed by human tumours (particularly by prostate, colon and pancreatic carcinomas, but also by interdigitating DCs), promotes resistance to immunemediated rejection of the tumour cells ${ }^{104}$. Locally produced IDO can block the proliferation of $\mathrm{CD}^{+} \mathrm{T}$ cells at the tumour site ${ }^{104}$ (FIG. 4C), as well as promote the apoptosis of $\mathrm{CD}^{+} \mathrm{T}$ cells ${ }^{105}$. Expression of CD95L by the tumour itself or by locally activated T cells might also induce the death of CD95-expressing tumour-specific $\mathrm{T}$ cells ${ }^{73}$.

In mouse models, advanced cancer invariably subverts immune function. Typically, tumour-specific CD ${ }^{+}$ $\mathrm{T}$ cells are activated at the stage of initiation of tumour growth, but these cells show a progressive loss of cytolytic function at the later stage of tumour expansion ${ }^{106}$. Similarly, tumour-specific CD $4^{+} \mathrm{T}$ cells progressively lose their antitumour activity ${ }^{107}$, whereas the number of $\mathrm{T}_{\text {Reg }}$ cells increases ${ }^{108}$. Indeed, the induction of tolerance to the tumour might even be a required factor for the initial steps of tumorigenesis ${ }^{13}$.

The exact molecular mechanisms by which tumours mediate immunosubversion are the subject of intense investigation. One possible explanation for how tumours subvert the immune response is to consider that the tumour is a 'false' lymphoid organ; therefore, $\mathrm{T}$-cell priming in the tumour microenvironment is defective as a result of the presence of dysfunctional or tolerogenic antigen-presenting cells ${ }^{109}$. Indeed, tumour beds contain various factors (such as VEGF, IL-6, IL-10, TGF $\beta$, macrophage colony-stimulating factor (M-CSF), NOS2, arginase-1, IDO, PGE 2 , COX2 and gangliosides) that can inhibit the differentiation, maturation and function of DCs ${ }^{109}$ (FIG. 4b,d,e). Accordingly, local DCs tend to mediate immunosuppressive, rather than immunostimulatory, effects and to promote $\mathrm{T}_{\text {Reg }}$-cell differentiation ${ }^{58}$. In an ultraviolet-irradiation-induced tumour model, irradiation-induced immunosuppression was found to be mediated by CD1d-restricted NKT cells. These CD4 ${ }^{+}$NKT cells produced IL-13, which suppressed CTL-mediated tumour rejection. Moreover, IL-13 from NKT cells activated myeloid suppressor cells to produce TGF $\beta$, which also suppressed CTL activity ${ }^{110}$ (FIG. 4g). In addition, tumour-associated macrophages mostly belong to the M2 class of macrophages, which produce arginase-1, IL-10, TGF $\beta$ and $\mathrm{PGE}_{2}$, and favour $\mathrm{T}_{\mathrm{H}}$ 2-cell responses (whereas $\mathrm{M} 1$ macrophages, which are tumoricidal, produce NOS2, IL-12 and lymphotoxin- $\alpha)^{111}$. Moreover, in patients 

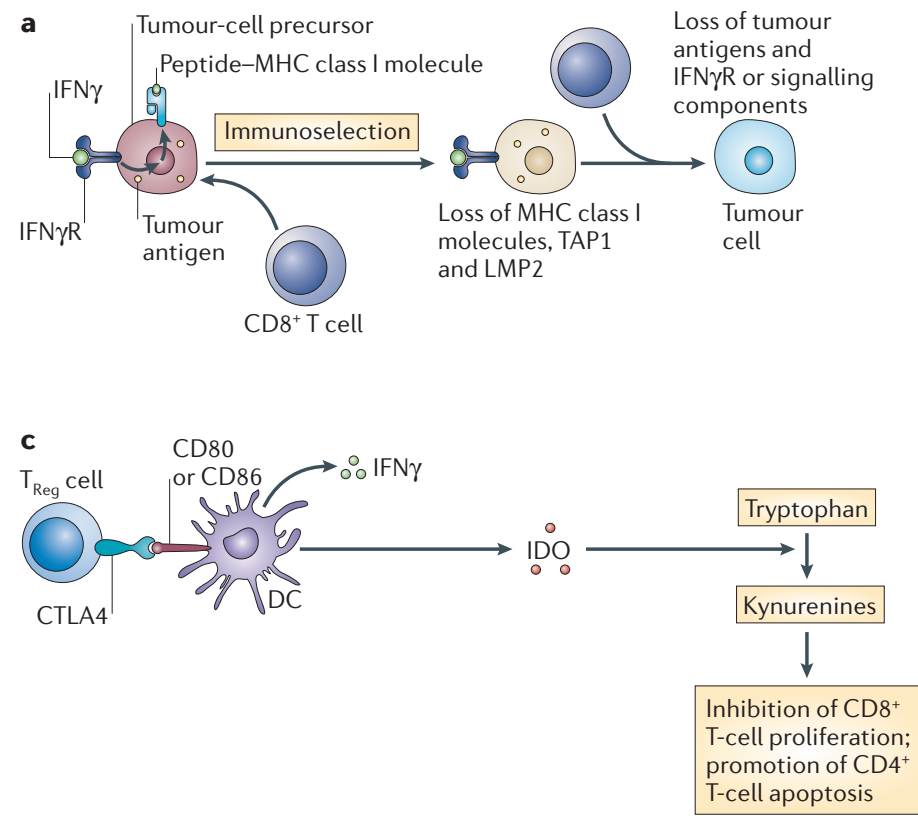

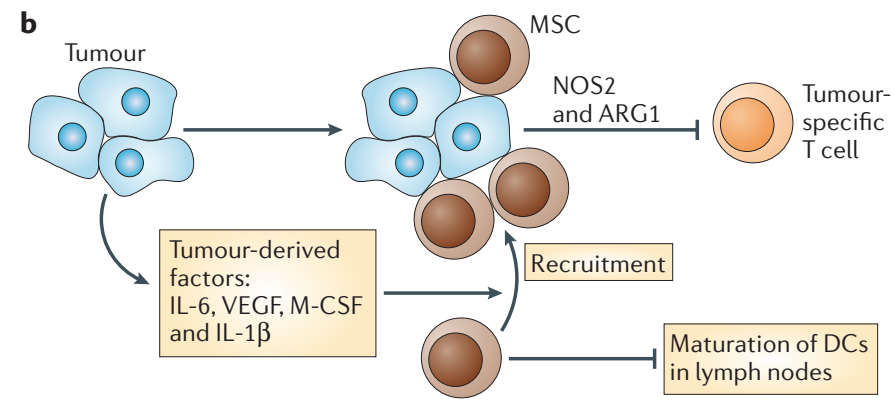

d

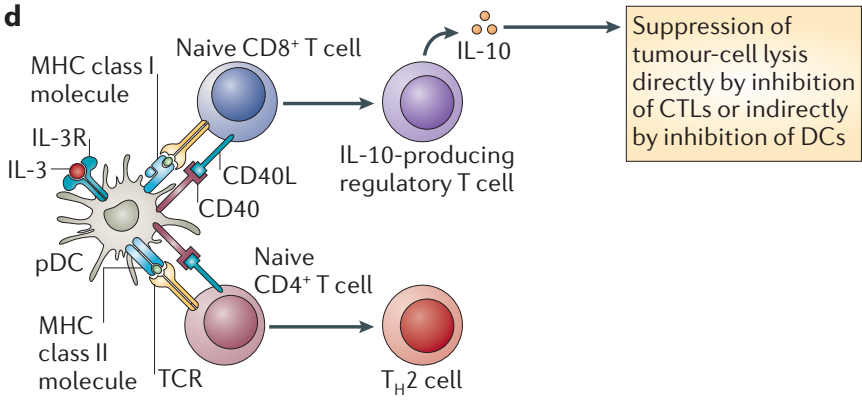

f
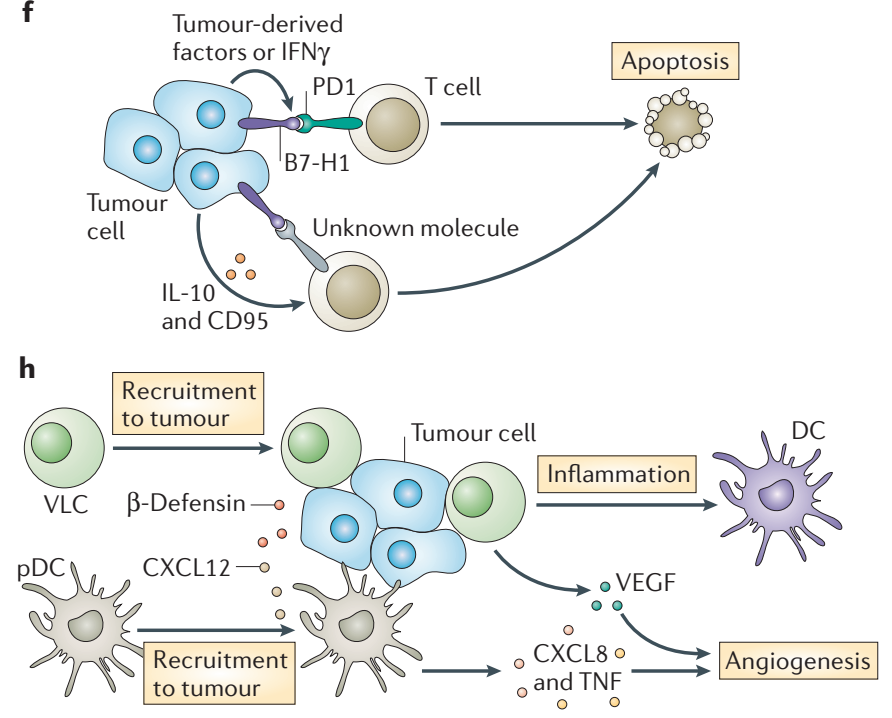

with immature DCs results in T-cell anergy, together with IL-10 production and IL-10-independent, cell-contact-dependent regulatory activity. f $\mid$ B7-H1 (and B7-H4) is expressed by some tumours (for example, in response to IFN $\gamma$ ), and it directly promotes T-cell apoptosis through programmed cell death 1 (PD1)-dependent pathways or PD1-independent pathways (which are mediated by IL-10 or CD95). $\mathbf{g}$ |CD4 ${ }^{+}$natural killer T (NKT) cells produce IL-13, which suppresses CTL-mediated tumour rejection through a pathway that involves the $\alpha$-chain of the IL-13 receptor (IL-13R $\alpha$ ) and STAT6. IL-13 produced by NKT cells can also activate MSCs to produce transforming growth factor- $\beta$ (TGF $\beta$ ), which suppresses CTLs. $\mathbf{h}$ | Vascular leukocyte cells (VLCs) and pDCs are attracted to tumour beds through $\beta$-defensins and CXC-chemokine ligand 12 (CXCL12), respectively. The subsequent angiogenic effect is mediated by CXCL8 in the case of pDCs and by vascular endothelial growth factor (VEGF) in the case of VLCs, which can differentiate into endothelial cells or into bona fide DCs during acute inflammation. APC, antigen-presenting cell; IFN $\gamma R$, IFN $\gamma$ receptor; IL-3R, IL-3 receptor; LMP2, low-molecular-mass protein 2; M-CSF, macrophage colonystimulating factor; TAP1, transporter associated with antigen processing 1 ; TCR, T-cell receptor; TNF, tumour-necrosis factor. 
with ovarian carcinoma, plasmacytoid DCs (pDCs) have been shown to induce the clonal expansion of IL-10-producing $\mathrm{CD}^{+}$regulatory T cells ${ }^{112}$ (FIG. $4 \mathrm{~d}$ ).

Another possible explanation for tumour-mediated immunosubversion is based on a quantitative argument. Tumour characteristics that are immunostimulatory in small tumours can become immunosuppressive in large tumours. For example, the expression of NKG2D ligands (which stimulates an immune response at the initial stages of oncogenesis, as discussed earlier) seems to be immunosuppressive in larger tumours. NKG2D-ligandexpressing tumour cells (as well as soluble NKG2D ligands that are shed from tumour cells) can downregulate NKG2D expression by $\mathrm{CD}^{+} \mathrm{T}$ cells and NK cells or can uncouple NKG2D signalling from intracellular mobilization of $\mathrm{Ca}^{2+}$ or cell-mediated cytolysis, thereby contributing to suppression of the immune response ${ }^{113}$. Similarly, it could be argued that large tumours cause a general or specific downregulation of T-cell responses as a result of 'high-dose tolerance' to tumour antigens. Following successful systemic chemotherapy - for example, for ovarian carcinoma $-\mathrm{CD} 8^{+} \mathrm{T}$-cell function can recover ${ }^{114}$, indicating that antitumour chemotherapies that have limited immunosuppressive side-effects can restore the normal immune response by abolishing tumour-mass-related immunosubversion. If this quantitative argument is correct, immunosubversion would be particularly important in advanced cancer.

In conclusion, it seems that there are numerous ways by which tumour cells can evade or 'paralyse' immunosurveillance. However, which of these multiple mechanisms affects oncogenesis and cancer progression in humans remains an open question.

Of note, in some cases, it is possible that, although an immune response to tumours is mounted, this response fails to eliminate the tumours or could even stimulate carcinogenesis and tumour progression, as a result of chronic inflammation. For example, in mouse models, $\mathrm{CD} 4^{+} \mathrm{T}$ cells have been shown to contribute to squamous-cell carcinogenesis induced by humanpapillomavirus antigens ${ }^{115}$. Moreover, transplantation of allogeneic haematopoietic cells has been shown to increase microsatellite instability in transformed epithelial cells from patients with squamous-cell cancer ${ }^{116}$. In patients with breast, prostate or bladder cancer, the secretion of IL-4 might upregulate the expression of anti-apoptotic proteins (such as FLIP and BCL- $\mathrm{X}_{\mathrm{L}}$ ) by the tumour ${ }^{117}$. Although cytotoxic agents produced by immune cells can mediate tumour destruction, this leads to the release of tumour-cell antigens, which can function as tissue-specific chemoattractants and promote leukocyte migration. For example, tumour antigens (or self antigens) - such as gp100 (glycoprotein 100), MUC1 and carcinoembryonic antigen - can attract immature DCs (for example, through binding of gp100 to CC-chemokine receptor 2) ${ }^{118}$, but tumour antigens cannot promote the maturation of immature DCs at these chemotactic concentrations. Such increased trafficking of undifferentiated immature DCs to tumour beds might accelerate tumour growth in the absence of maturation stimuli $^{119}$. Vascular leukocyte cells (VLCs) and pDCs can be recruited to tumour beds by pro-inflammatory mediators - such as $\beta$-defensins and CXC-chemokine ligand 12 (CXCL12), respectively - and contribute to angiogenesis. VLCs contribute to angiogenesis by differentiating into endothelial cells in the presence of local VEGF, and pDCs contribute by secreting the angiogenic cytokines TNF and CXCL8 (also known as IL-8) ${ }^{120,121}$ (FIG. 4h). These examples illustrate (without clarifying how) that an incomplete antitumour immune response might benefit the tumour but not the host.

\section{Concluding remarks}

Clinical oncologists tend to conceive of, and treat, cancer as a cell-intrinsic phenomenon, ignoring the contribution of the innate and adaptive immune systems to the therapeutic response. Given the data that we review here, it is plausible that evasion or subversion of cancer immunosurveillance is a central hallmark of many malignancies. It can be anticipated that progress in molecular profiling of cancers and in individually applied pharmacogenetic and immunogenetic approaches will facilitate patients' understanding of the cancer-immunosurveillance system. Comprehensive information on the tumour and the immune status of an individual could be expected to provide a precise picture of the ongoing evolution of the tumour (and therefore a useful tool for prognostic extrapolation), as well as to yield invaluable information about which strategy (surgery, chemotherapy, radiotherapy and/or immunotherapy) will result in an optimal therapeutic outcome.

Owing to the mainly nonspecific cytostatic and cytotoxic effects, most current chemotherapeutic regimens are immunosuppressive. However, some chemotherapeutic agents can stimulate an antitumour immune response, by having 'side-effects' on the immune system. For example, low doses of cyclophosphamide can selectively deplete and inhibit $\mathrm{T}_{\text {Reg }}$ cells, thereby restoring normal CTL and NK-cell function in patients with cancer ${ }^{122,123}$. Similarly, imatinib mesylate can stimulate the activity of NK cells, correlating with a favourable clinical outcome for patients with gastrointestinal stromal tumours ${ }^{124}$. Other chemotherapeutic agents can increase the antigenic properties of tumour cells. Therefore, intratumoral injections of anthracyclines and local irradiation of established tumours can promote immunogenic tumour-cell death ${ }^{82}$. Several cytotoxic drugs can stimulate tumour-cell-surface expression of proteins that facilitate tumour-cell lysis or tumour-cell recognition. As an example, numerous DNA-damaging agents induce the expression of death receptors such as TRAIL receptor 1 (TRAILR1), TRAILR2 and CD95 by tumour cells, thereby facilitating the lysis of these cells by TRAIL- or CD95L-expressing immune effector cells ${ }^{125}$. In addition, radiotherapy increases the expression of MHC class I molecules and improves antigen presentation $^{126}$. Also, histone-deacetylase inhibitors induce hepatocellular-carcinoma cells to express NKG2D ligands (such as MICA and MICB), thereby increasing the lysis of these cells by NK cells ${ }^{127}$. It is our hope that similar therapeutic strategies will improve the effcacy of antitumour therapies, especially if they are combined with immunostimulatory regimens. 
1. Hanahan, D. \& Weinberg, R. A. The hallmarks of cancer. Cell 100, 57-70 (2000). This landmark review was the first to enumerate systematically the cell-intrinsic characteristics of cancer cells.

2. Dunn, G. P., Old, L. J. \& Schreiber, R. D. The three Es of cancer immunoediting. Annu. Rev. Immunol. 22, 329-360 (2004).

This was the first paper to propose that avoidance of immunosurveillance could be the seventh hallmark of cancer.

3. Smyth, M. J., Dunn, G. P. \& Schreiber, R. D. Cancer immunosurveillance and immunoediting: the roles of immunity in suppressing tumor development and shaping tumor immunogenicity. Adv. Immunol. 90, 1-50 (2006)

4 Dunn, G. P., Old, L. J. \& Schreiber, R. D. The immunobiology of cancer immunosurveillance and immunoediting. Immunity 21, 137-148 (2004). This paper brilliantly summarizes the concept of immunoediting.

5. Alfons, B. et al. Enhancement of experimental allergic encephalomyelitis in mice by antibodies against IFN- $\gamma$ J. Immunol. 140, 1506-1510 (1988).

6. Takeda, K. et al. Critical role for tumor necrosis factorrelated apoptosis-inducing ligand in immune surveillance against tumor development. J. Exp. Med. 195, 161-169 (2002).

A pioneering study that underscores the role of

TRAIL in cancer immunosurveillance.

7. Raulet, D. H. Roles of the NKG2D immunoreceptor and its ligands. Nature Rev. Immunol. 3, 781-790 (2003).

8. Smyth, M. J. et al. Sequential activation of NKT cells and NK cells provides effective innate immunotherapy of cancer. J. Exp. Med. 201 1973-1985 (2005).

9. Taieb, J. et al. A novel dendritic cell subset involved in tumor immunosurveillance. Nature Med. 12, 214-219 (2006)

10. Dhodapkar, M. V., Krasovsky, J., Osman, K. \& Geller, M. D. Vigorous premalignancy-specific effector T cell response in the bone marrow of patients with monoclonal gammopathy. J. Exp. Med. 198, 1753-1757 (2003).

11. Beckhove, P. et al. Specifically activated memory T cell subsets from cancer patients recognize and reject xenotransplanted autologous tumors. J. Clin. Invest. 114, 67-76 (2004).

12. Schmitz-Winnenthal, F. H. et al. High frequencies of functional tumor-reactive $T$ cells in bone marrow and blood of pancreatic cancer patients. Cancer Res. 65, 10079-10087 (2005)

13. Willimsky, G. \& Blankenstein, T. Sporadic immunogenic tumours avoid destruction by inducing T-cell tolerance. Nature 437, 141-146 (2005). An elegant study showing that immunoediting might not occur for spontaneously arising tumours that overexpress simian virus $\mathbf{4 0}$ (SV40).

14. Wang, X. et al. Autoantibody signatures in prostate cancer. N. Engl. J. Med. 353, 1224-1235 (2005).

15. Suzuki, H., Graziano, D. F., McKolanis, J. \& Finn, O. J. $\mathrm{T}$ cell-dependent antibody responses against aberrantly expressed cyclin B1 protein in patients with cancer and premalignant disease. Clin. Cancer Res. 11, 1521-1526 (2005)

16. Li, Y. et al. p53 autoantibodies predict subsequent development of cancer. Int. J. Cancer 114, 157-160 (2005).

17. Egloff, A. M., Weissfeld, J., Land, S. R. \& Finn, O. J. Evaluation of anticyclin B1 serum antibody as a diagnostic and prognostic biomarker for lung cancer. Ann. NY Acad. Sci. 1062, 29-40 (2005).

18. Zitvogel, L., Casares, N., Pequignot, M., Albert, M. L. $\&$ Kroemer, G. The immune response against dying tumor cells. Adv. Immunol. 84, 131-179 (2004).

19. Pages, F. et al. Epstein-Barr virus nuclear antigen 2 induces interleukin-18 receptor expression in B cells. Blood 105, 1632-1639 (2005).

20. Nakamura, E et al Genetic polymorphisms of the interleukin- 4 receptor $\alpha$ gene are associated with an increasing risk and a poor prognosis of sporadic rena cell carcinoma in a Japanese population. Clin. Cancer Res. 8, 2620-2625 (2002).

21. Curiel, T. J. et al. Specific recruitment of regulatory $\mathrm{T}$ cells in ovarian carcinoma fosters immune privilege and predicts reduced survival. Nature Med. 10 942-949 (2004)

22. Viguier, M. et al. Foxp3 expressing $\mathrm{CD} 4{ }^{+} \mathrm{CD} 25^{\text {high }}$ regulatory $T$ cells are overrepresented in human metastatic melanoma lymph nodes and inhibit the function of infiltrating T cells. J. Immunol. 173,

1444-1453 (2004).

23. Ghiringhelli, F. et al. $\mathrm{CD} 4{ }^{+} \mathrm{CD} 25^{+}$regulatory $\mathrm{T}$ cells inhibit natural killer cell functions in a transforming growth factor- $\beta$-dependent manner. J. Exp. Med. 202. 1075-1085 (2005)

References 21-23 provide strong evidence of a role for $T_{\text {Reg }}$ cells in tumour-induced tolerance.

24. Bronte, V. et al. Boosting antitumor responses of $T$ lymphocytes infiltrating human prostate cancers. J. Exp. Med. 201, 1257-1268 (2005).

25. Penn, I. Post-transplant malignancy: the role of immunosuppression. Drug Saf. 23, 101-113 (2000)

26. Hollenbeak, C. S. et al. Increased incidence of melanoma in renal transplantation recipients. Cancer 104, 1962-1967 (2005).

27. Kobayashi, N. Malignant neoplasms in registered cases of primary immunodeficiency syndrome. Jpn J. Clin. Oncol. 15 (Suppl. 1), 307-312 (1985).

28. Clementi, R et al. A proportion of patients with lymphoma may harbor mutations of the perforin gene. Blood 105, 4424-4428 (2005)

29. Sun, T. et al. FASL-844C polymorphism is associated with increased activation-induced $\mathrm{T}$ cell death and risk of cervical cancer. J. Exp. Med. 202, 967-974 (2005)

30. Carrington, M. et al. Hierarchy of resistance to cervical neoplasia mediated by combinations of killer immunoglobulin-like receptor and human leukocyte antigen loci. J. Exp. Med. 201, 1069-1075 (2005)

31. Nakachi, K., Hayashi, T., Imai, K. \& Kusunoki, Y. Perspectives on cancer immuno-epidemiology. Cancer Sci. 95, 921-929 (2004).

32. Imai, K., Matsuyama, S., Miyake, S., Suga, K. \& Nakachi, K. Natural cytotoxic activity of peripheralblood lymphocytes and cancer incidence: an 11-year follow-up study of a general population. Lancet 356 1795-1799 (2000)

33. Hayashi, T. et al. Identification of the NKG2D haplotypes associated with natural cytotoxic activity of peripheral blood lymphocytes and cancer immunosurveillance. Cancer Res. 66, 563-570 (2006)

References 31-33 introduce the novel concept of two hits for cancer development: first, the oncogenic event that leads to cell-surface expression of stress-associated molecules; and second, the recognition of these molecules by innate immune cells that display receptors with a polymorphism that allows tumour escape.

34. Lowe, S. W., Cepero, E. \& Evan, G. Intrinsic tumour suppression. Nature 432, 307-315 (2004)

35. Bartkova, J. et al. DNA damage response as a candidate anti-cancer barrier in early human tumorigenesis. Nature 434, 864-870 (2005).

36. Gorgoulis, V. G. et al. Activation of the DNA damage checkpoint and genomic instability in human precancerous lesions. Nature 434, 907-913 (2005) References 35 and 36 underscore the importance of the oncogene-induced ATM-CHK1-p53 pathway in the control of early pre-malignant lesions as a mechanism of tumour suppression.

37. Gasser, S., Orsulic, S., Brown, E. J. \& Raulet, D. H. The DNA damage pathway regulates innate immune system ligands of the NKG2D receptor. Nature 436, 1186-1190 (2005)

This seminal study provided the first indication that the DNA-damage response induced by endogenous stress (in this case, oncogene activation) or therapy is linked to the induction of expression of 'danger' signals.

38. Smyth, M. J. et al. NKG2D function protects the host from tumor initiation. J. Exp. Med. 202, 583-588 (2005)

39. Zhou, H. et al. DNA-based vaccines activate innate and adaptive antitumor immunity by engaging the NKG2D receptor. Proc. Natl Acad. Sci. USA 102 10846-10851 (2005)

40. Watson, N. F. et al. Expression of the stress-related $\mathrm{MHC}$ class I chain-related protein MICA is an indicator of good prognosis in colorectal cancer patients. Int. J. Cancer 118, 1445-1452 (2006).

41. Holdenrieder, S. et al. Soluble MICA in malignant diseases. Int. J. Cancer 118, 684-687 (2006).

42. Oppenheim, D. E. et al. Sustained localized expression of ligand for the activating NKG2D receptor impairs natural cytotoxicity in vivo and reduces tumor immunosurveillance. Nature Immunol. 6, 928-937 (2005)

An elegant study showing the immunosuppressive role of NKG2D ligands on NK-cell functions in mice.
43. Chen, Z. et al. Crucial role of p53-dependent cellular senescence in suppression of Pten-deficien tumorigenesis. Nature 436, 725-730 (2005)

44. Braig, M. et al. Oncogene-induced senescence as an initial barrier in lymphoma development. Nature $\mathbf{4 3 6}$ 660-665 (2005)

45. Pardoll, D. M. Spinning molecular immunology into successful immunotherapy. Nature Rev. Immunol. 2, 227-238 (2002).

46. Collado, M. et al. Tumour biology: senescence in premalignant tumours. Nature 436, 642 (2005)

47. Dunn, G. P. et al. A critical function for type interferons in cancer immunoediting. Nature Immunol. 6, 722-729 (2005).

48. Takaoka, A. et al. Integration of interferon- $\alpha / \beta$ signalling to $\mathrm{p} 53$ responses in tumour suppression and antiviral defence. Nature 424, 516-523 (2003).

49. Chaturvedi, V., Bodner, B., Qin, J. Z. \& Nickoloff, B. J. Knock down of p53 levels in human keratinocytes increases susceptibility to type I and type II interferoninduced apoptosis mediated by a TRAIL dependent pathway. J. Dermatol. Sci. 41, 31-41 (2006)

50. Stassi, G. et al. Thyroid cancer resistance to chemotherapeutic drugs via autocrine production of interleukin-4 and interleukin-10. Cancer Res. 63, 6784-6790 (2003).

51. Wang, T. et al. Regulation of the innate and adaptive immune responses by Stat-3 signaling in tumor cells. Nature Med. 10, 48-54 (2004).

52. Nabarro, S. et al. Coordinated oncogenic transformation and inhibition of host immune responses by the PAX3-FKHR fusion oncoprotein J. Exp. Med. 202, 1399-1410 (2005).

53. Kryczek, I. et al. B7-H4 expression identifies a novel suppressive macrophage population in human ovarian carcinoma. J. Exp. Med. 203, 871-881 (2006).

54. Pupa, S. M., Tagliabue, E., Menard, S. \& Anichini, A. HER-2: a biomarker at the crossroads of breast cancer immunotherapy and molecular medicine. J. Cell. Physiol. 205, 10-18 (2005)

55. Carbone, D. P. et al. Immunization with mutant p53 and K-ras-derived peptides in cancer patients: immune response and clinical outcome. J. Clin. Oncol. 23 5099-5107 (2005).

56. Muraoka-Cook, R. S., Dumont, N. \& Arteaga, C. L. Dual role of transforming growth factor $\beta$ in mammary tumorigenesis and metastatic progression. Clin Cancer Res. 11, 937s-943s (2005).

57 Gorelik, L \& Flavell, R. A. Transforming growth factor- $\beta$ in T-cell biology. Nature Rev. Immunol. 2, 46-53 (2002)

58. Ghiringhelli, F. et al. Tumor cells convert immature myeloid dendritic cells into TGF- $\beta$-secreting cells inducing $\mathrm{CD} 4{ }^{+} \mathrm{CD} 25^{+}$regulatory $\mathrm{T}$ cell proliferation. J. Exp. Med. 202, 919-929 (2005).

59. Bettelli, E. et al. Reciprocal developmental pathways for the generation of pathogenic effector $T_{H} 17$ and regulatory T cells. Nature 441, 235-238 (2006).

60 Zhang $\mathrm{O}$ et al. Adoptive transfer of tumor-reactive transforming growth factor- $\beta$-insensitive $C D 8^{+} \mathrm{T}$ cells eradication of autologous mouse prostate cancer. Cancer Res. 65, 1761-1769 (2005).

61. Green, D. R. \& Kroemer, G. The pathophysiology of mitochondrial cell death. Science 305, 626-629 (2004).

62. Debatin, K. M. \& Krammer, P. H. Death receptors in chemotherapy and cancer. Oncogene 23, 2950-2966 (2004).

63. Kroemer, G. \& Martin, S. J. Caspase-independent cell death. Nature Med. 11, 725-730 (2005).

64. Ravi, R. et al. Resistance of cancers to immunologic cytotoxicity and adoptive immunotherapy via X-linked inhibitor of apoptosis protein expression and coexisting defects in mitochondrial death signaling. Cancer Res. 66, 1730-1739 (2006).

65. Liu, K., Caldwell, S. A. \& Abrams, S. I. Immune selection and emergence of aggressive tumor variants as negative consequences of Fas-mediated cytotoxicity and altered IFN- $\gamma$-regulated gene expression. Cancer Res. 65, 4376-4388 (2005).

66. Andersen, M. H., Reker, S., Kvistborg, P. Becker, J. C. \& thor Straten, P. Spontaneous immunity against $\mathrm{Bcl}-\mathrm{x}_{\mathrm{L}}$ in cancer patients. J. Immunol. 175, 2709-2714 (2005)

67. Ren, J. et al. MUC1 oncoprotein is targeted to mitochondria by heregulin-induced activation of c-Src and the molecular chaperone HSP90. Oncogene 25, 20-31 (2006)

68. Monti, P. et al. Tumor-derived MUC1 mucins interact with differentiating monocytes and induce 
IL-10 $0^{\text {high }}$ L- $12^{\text {low }}$ regulatory dendritic cell. J. Immunol. 172, 7341-7349 (2004).

69. Carlos, C. A. et al. Human tumor antigen MUC1 is chemotactic for immature dendritic cells and elicits maturation but does not promote $\mathrm{T}_{H} 1$ type immunity. J. Immunol. 175, 1628-1635 (2005).

70. Dohi, T., Beltrami, E., Wall, N. R., Plescia, J. \& Altieri, D. C. Mitochondrial survivin inhibits apoptosis and promotes tumorigenesis. J. Clin. Invest 114, 1117-1127 (2004).

71. Andersen, M. H., Pedersen, L. O., Becker, J. C. \& Straten, P. T. Identification of a cytotoxic T lymphocyte response to the apoptosis inhibitor protein survivin in cancer patients. Cancer Res. 61, 869-872 (2001).

72. Asanuma, K., Tsuji, N., Endoh, T., Yagihashi, A. \& Watanabe, N. Survivin enhances Fas ligand expression via up-regulation of specificity protein 1-mediated gene transcription in colon cancer cells. J. Immunol. 172, 3922-3929 (2004)

73. Igney, F. H. \& Krammer, P. H. Tumor counterattack: fact or fiction? Cancer Immunol. Immunother. $\mathbf{5 4}$ 1127-1136 (2005).

74. Wajant, H. CD95L/FasL and TRAIL in tumour surveillance and cancer therapy. Cancer Treat. Res. 130, 141-165 (2006)

75. Bennett, M. W. et al. The Fas counterattack in vivo: apoptotic depletion of tumor-infiltrating lymphocytes associated with Fas ligand expression by human esophageal carcinoma. J. Immunol. 160, 5669-5675 (1998).

76. Okada, K. et al. Frequency of apoptosis of tumorinfiltrating lymphocytes induced by Fas counterattack in human colorectal carcinoma and its correlation with prognosis. Clin. Cancer Res. 6, 3560-3564 (2000)

77. Scott, C. L. et al. Apaf-1 and caspase-9 do not act as tumor suppressors in myc-induced lymphomagenesis or mouse embryo fibroblast transformation. J. Cell Biol. 164, 89-96 (2004).

78. Tanimoto, T. et al. Nuclear expression of ClAP-1, an apoptosis inhibiting protein, predicts lymph node metastasis and poor patient prognosis in head and neck squamous cell carcinomas. Cancer Lett. 224, 141-151 (2005)

79. Tamm, I. et al. XIAP expression correlates with monocytic differentiation in adult de novo AML: impact on prognosis. Hematol. J. 5, 489-495 (2004)

80. Soengas, M. S. et al. Inactivation of the apoptosis effector Apaf- 1 in malignant melanoma. Nature 409 207-211 (2001)

81. Umetani, N et al. Allelic imbalance of APAF- 1 locus at $12 q 23$ is related to progression of colorecta carcinoma. Oncogene 23, 8292-8300 (2004)

82. Casares, N. et al. Caspase-dependent immunogenicity of doxorubicin-induced tumor cell death. J. Exp. Med. 202, 1691-1701 (2005)

This article provides evidence that the immunogenicity of some chemotherapy protocols is influenced by the propensity of tumour cells to activate caspases.

83. Nikitina, E. Y. et al. Versatile prostate cancer treatment with inducible caspase and interleukin-12. Cancer Res. 65, 4309-4319 (2005).

84. Amarnath, S. M. et al. In vitro quantification of the cytotoxic $T$ lymphocyte response against human telomerase reverse transcriptase in breast cancer. Int. J. Oncol. 25, 211-217 (2004).

85. Maecker, B. et al. Rare naturally occurring immune responses to three epitopes from the widely expressed tumour antigens hTERT and CYP1B1 in multiple myeloma patients. Clin. Exp. Immunol. 141, 558-562 (2005).

86. Ichiki, Y. et al. Simultaneous cellular and humoral immune response against mutated p53 in a patient with lung cancer. J. Immunol. 172, 4844-4850 (2004).

87. Della Porta, M. et al. Dendritic cells and vascular endothelial growth factor in colorectal cancer: correlations with clinicobiological findings. Oncology 68, 276-284 (2005)

88. Ohm, J. E. et al. VEGF inhibits T-cell development and may contribute to tumor-induced immune suppression. Blood 101, 4878-4886 (2003).

89. Yang, L. \& Carbone, D. P. Tumor-host immune interactions and dendritic cell dysfunction. $A d v$. Cancer Res. 92, 13-27 (2004)

90. Brown, J. R. \& DuBois, R. N. COX-2: a molecular target for colorectal cancer prevention. J. Clin. Oncol. 23, 2840-2855 (2005).

91. Stolina, M. et al. Specific inhibition of cyclooxygenase 2 restores antitumor reactivity by altering the balance of IL-10 and IL-12 synthesis. J. Immunol. 164, 361-370 (2000).

92. Lang, S. et al. Impaired monocyte function in cancer patients: restoration with a cyclooxygenase- 2 inhibitor. FASEB J. 17, 286-288 (2003).

93. Boles, K. S., Barchet, W., Diacovo, T., Cella, M. \& Colonna, M. The tumor suppressor TSLC1/NECL-2 triggers NK-cell and CD8 $8^{+}$T-cell responses through the cell-surface receptor CRTAM. Blood 106, 779-786 (2005).

94. Kroemer, G. \& Jaattela, M. Lysosomes and autophagy in cell death control. Nature Rev. Cancer 5, 886-897 (2005)

95. Calderwood, S. K., Theriault, J. R. \& Gong, J. Message in a bottle: role of the 70-kDa heat shock protein family in anti-tumor immunity. Eur. J. Immunol. 35 2518-2527 (2005)

96. Binder, R. J. \& Srivastava, P. K. Peptides chaperoned by heat-shock proteins are a necessary and sufficient source of antigen in the cross-priming of $\mathrm{CD} 8{ }^{+} \mathrm{T}$ cells. Nature Immunol. 6, 593-599 (2005).

97. Gastpar, R. et al. Heat shock protein 70 surfacepositive tumor exosomes stimulate migratory and cytolytic activity of natural killer cells. Cancer Res. 65 , 5238-5247 (2005)

98. Stefanidakis, M. \& Koivunen, E. Cell-surface association between matrix metalloproteinases: role of the complexes in leukocyte migration and cancer progression. Blood 108, 1441-1450 (2006).

99. So, T. et al. Haplotype loss of HLA class I antigen as an escape mechanism from immune attack in lung cancer. Cancer Res. 65, 5945-5952 (2005)

100. Atkins, D. et al. MHC class I antigen processing pathway defects, ras mutations and disease stage in colorectal carcinoma. Int. J. Cancer 109, 265-273 (2004).

101. Medema, J. P. et al. Blockade of the granzyme B/ perforin pathway through overexpression of the serine protease inhibitor PI-9/SPI-6 constitutes a mechanism for immune escape by tumors. Proc. Natl Acad. Sci. USA 98, 11515-11520 (2001)

102. Ochsenbein, A. F. Immunological ignorance of solid tumors. Springer Semin. Immunopathol. 27, 19-35 (2005)

103. Bronte, V. \& Zanovello, P. Regulation of immune responses by L-arginine metabolism. Nature Rev. Immunol. 5, 641-654 (2005).

A stimulating review about the characterization of myeloid suppressor cells.

104. Uyttenhove, C. et al. Evidence for a tumoral immune resistance mechanism based on tryptophan degradation by indoleamine 2,3-dioxygenase. Nature Med. 9, 1269-1274 (2003).

105. Terness, P. et al. Inhibition of allogeneic T cell proliferation by indoleamine 2,3-dioxygenase expressing dendritic cells: mediation of suppression by tryptophan metabolites. J. Exp. Med. 196, 447-457 (2002).

106. Huang, Y., Obholzer, N., Fayad, R. \& Qiao, L. Turning on/off tumor-specific CTL response during progressive tumor growth. J. Immunol. 175, 3110-3116 (2005).

107. Zhou, G., Lu, Z., McCadden, J. D., Levitsky, H. I. \& Marson, A. L. Reciprocal changes in tumor antigenicity and antigen-specific $T$ cell function during tumor progression. J. Exp. Med. 200, 1581-1592 (2004).

108. Sakaguchi, S. Naturally arising $\mathrm{CD} 4^{+}$regulatory $\mathrm{T}$ cells for immunologic self-tolerance and negative control of immune responses. Annu. Rev. Immunol. 22, 531-562 (2004)

109. Zou, W. Immunosuppressive networks in the tumour environment and their therapeutic relevance. Nature Rev. Cancer 5, 263-274 (2005).

110. Terabe, M. \& Berzofsky, J. A. Immunoregulatory T cells in tumor immunity. Curr. Opin. Immunol. 16, 157-162 (2004).

An overview of the suppressive role of IL-13 produced by NKT cells during tolerance induced by irradiation with ultraviolet light. This suppressive process involves myeloid suppressor cells and TGF $\beta$.

111. Guiducci, C., Vicari, A. P., Sangaletti, S., Trinchieri, G. $\&$ Colombo, M. P. Redirecting in vivo elicited tumor infiltrating macrophages and dendritic cells towards tumor rejection. Cancer Res. 65, 3437-3446 (2005)

112. Wei, S. et al. Plasmacytoid dendritic cells induce $C D 8^{+}$ regulatory $\mathrm{T}$ cells in human ovarian carcinoma. Cancer Res. 65, 5020-5026 (2005).
113. Coudert, J. D. et al. Altered NKG2D function in NK cells induced by chronic exposure to NKG2D ligandexpressing tumor cells. Blood 106, 1711-1717 (2005).

114. Coleman, S. et al. Recovery of CD8 ${ }^{+}$T-cell function during systemic chemotherapy in advanced ovarian cancer. Cancer Res. 65, 7000-7006 (2005).

115. Daniel, D. et al. Immune enhancement of skin carcinogenesis by $\mathrm{CD}^{+}{ }^{+} \mathrm{T}$ cells. J. Exp. Med. 197, 1017-1028 (2003).

116. Faber, P. et al. Frequent genomic alterations in epithelium measured by microsatellite instability following allogeneic hematopoietic cell transplantation in humans. Blood 107, 3389-3396 (2005).

117. Conticello, C. et al. IL-4 protects tumor cells from anti-CD95 and chemotherapeutic agents via up-regulation of antiapoptotic proteins. J. Immunol. 172, 5467-5477 (2004).

118. Oppenheim, J. J. et al. Autoantigens act as tissue-specific chemoattractants. J. Leukoc. Biol. 77, 854-861 (2005)

119. Bonnotte, B., Crittenden, M., Larmonier, N., Gough, M. \& Vile, R. G. MIP-3 $\alpha$ transfection into a rodent tumor cell line increases intratumoral dendritic cell infiltration but enhances (facilitates) tumor growth and decreases immunogenicity. J. Immunol. 173, 4929-4935 (2004).

120. Curiel, T. J. et al. Dendritic cell subsets differentially regulate angiogenesis in human ovarian cancer Cancer Res. 64, 5535-5538 (2004).

121. Coukos, G., Benencia, F., Buckanovich, R. J. \& Conejo-Garcia, J. R. The role of dendritic cell precursors in tumour vasculogenesis. Br. J. Cancer 92, 1182-1187 (2005)

122. Berd, D. \& Mastrangelo, M. J. Effect of low dose cyclophosphamide on the immune system of cancer patients: reduction of T-suppressor function without depletion of the $\mathrm{CD}^{+}{ }^{+}$subset. Cancer Res. 47, 3317-3321 (1987)

123. Ghiringhelli, F. et al. Metronomic cyclophosphamide regimen selectively depletes $C D 4+C D 25+$ regulatory $\mathrm{T}$ cells and restores $\mathrm{T}$ and NK effector functions in end stage cancer patients. Cancer Immunol. Immunother. (in the press)

124. Borg, C. et al. Novel mode of action of c-kit tyrosine kinase inhibitors leading to NK cell-dependent antitumor effects. J. Clin. Invest. 114, 379-388 (2004).

The first study to show the side-effect of imatinib mesylate on the immune system of mice and patients

125. Fulda, S. \& Debatin, K. M. Targeting apoptosis pathways in cancer therapy. Curr. Cancer Drug Targets 4, 569-576 (2004).

126. Reits, E. A. et al. Radiation modulates the peptide repertoire, enhances MHC class I expression, and induces successful antitumor immunotherapy. J. Exp. Med. 203, 1259-1271 (2006).

127. Armeanu, S. et al. Natural killer cell-mediated lysis of hepatoma cells via specific induction of NKG2D ligands by the histone deacetylase inhibitor sodium valproate. Cancer Res. 65, 6321-6329 (2005).

\section{Acknowledgements}

The authors are supported by grants from the European Union, the Ligue Nationale contre le Cancer (France), Cancéropôle Ille-de-France (France) and the Institut National du Cancer (France).

\section{Competing interests statement}

The authors declare no competing financial interests.

DATABASES

The following terms in this article are linked online to: Entrez Gene:

http://www.ncbi.nlm.nih.gov/entrez/query.fcgi?db=gene APAF1 | arginase-1 | ATM | BCL-2 |BCL-X | CD95 |CD95L | CHK1 |COX2 | CXCL8 | CXCL12 |EGFR |ERBB2 |IDO | IFN $\gamma$ | IL-4 | IL-6 | IL-10| IL-13 |LMP2 | MCL1 | M-CSF | MUC1 | NKG2D | NOS2 | p53 | TAP1 | TGF 3 | TNF | TRAIL | TSLC1 | VEGF FURTHER INFORMATION Institut Gustave-Roussy: www.igr.fr Access to this links box is available online. 